\title{
The 400d Galaxy Cluster Survey weak lensing programme
}

\section{Evidence for consistent $W L$ and $X$-ray masses at $z \approx 0.5^{\star}$}

\author{
Holger Israel ${ }^{1,2}$, Thomas H. Reiprich ${ }^{2}$, Thomas Erben ${ }^{2}$, Richard J. Massey ${ }^{1}$, Craig L. Sarazin ${ }^{3}$, \\ Peter Schneider ${ }^{2}$, and Alexey Vikhlinin ${ }^{4}$ \\ ${ }^{1}$ Department of Physics, Durham University, South Road, Durham DH1 3LE, UK \\ e-mail: holger.israel@durham.ac.uk \\ 2 Argelander-Institut für Astronomie, Auf dem Hügel 71, 53121 Bonn, Germany \\ 3 Department of Astronomy, University of Virginia, 530 McCormick Road, Charlottesville VA 22904, USA \\ ${ }^{4}$ Harvard-Smithsonian Center for Astrophysics, 60 Garden Street, Cambridge MA 02138, USA
}

Received 17 October 2013 / Accepted 11 February 2014

\begin{abstract}
Context. Scaling properties of galaxy cluster observables with cluster mass provide central insights into the processes shaping clusters. Calibrating proxies for cluster mass that are relatively cheap to observe will moreover be crucial to harvest the cosmological information available from the number and growth of clusters with upcoming surveys like eROSITA and Euclid. The recent Planck results led to suggestions that X-ray masses might be biased low by $\sim 40 \%$, more than previously considered.

Aims. We aim to extend knowledge of the weak lensing - X-ray mass scaling towards lower masses (as low as $1 \times 10^{14} M_{\odot}$ ) in a sample representative of the $z \sim 0.4-0.5$ population. Thus, we extend the direct calibration of cluster mass estimates to higher redshifts.

Methods. We investigate the scaling behaviour of MMT/Megacam weak lensing (WL) masses for 8 clusters at $0.39 \leq z \leq 0.80$ as part of the 400d WL programme with hydrostatic Chandra X-ray masses as well as those based on the proxies, e.g. $Y_{\mathrm{X}}=T_{\mathrm{X}} M_{\mathrm{gas}}$.

Results. Overall, we find good agreement between WL and X-ray masses, with different mass bias estimators all consistent with zero. When subdividing the sample into a low-mass and a high-mass subsample, we find the high-mass subsample to show no significant mass bias while for the low-mass subsample, there is a bias towards overestimated X-ray masses at the $\sim 2 \sigma$ level for some mass proxies. The overall scatter in the mass-mass scaling relations is surprisingly low. Investigating possible causes, we find that neither the greater range in WL than in X-ray masses nor the small scatter can be traced back to the parameter settings in the WL analysis. Conclusions. We do not find evidence for a strong ( $40 \%)$ underestimate in the X-ray masses, as suggested to reconcile recent Planck cluster counts and cosmological constraints. For high-mass clusters, our measurements are consistent with other studies in the literature. The mass dependent bias, significant at $\sim 2 \sigma$, may hint at a physically different cluster population (less relaxed clusters with more substructure and mergers); or it may be due to small number statistics. Further studies of low-mass high- $z$ lensing clusters will elucidate their mass scaling behaviour.
\end{abstract}

Key words. galaxies: clusters: general - cosmology: observations - gravitational lensing: weak - X-rays: galaxies: clusters

\section{Introduction}

Galaxy cluster masses hold a crucial role in cosmology. In the paradigm of hierarchical structure formation from tiny fluctuations in the highly homogeneous early cosmos after inflation, clusters emerge via the continuous matter accretion onto local minima of the gravitational potential. Depending sensitively on cosmological parameters, the cluster mass function, i.e. their abundance as function of mass and redshift $z$, provides observational constraints to cosmology (e.g., Vikhlinin et al. 2009b; Allen et al. 2011; Planck Collaboration XX 2014).

Observers use several avenues to determine cluster masses: properties of the X-ray-emitting intracluster medium (ICM), its imprint on the cosmic microwave background via the SunyaevZel'dovich (SZ) effect in the sub-mm regime, galaxy richness estimates and dynamical masses via optical imaging and

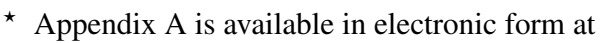
http: //www . aanda.org spectroscopy, and gravitational lensing. Across all wavelengths, cluster cosmology surveys are under preparation, aiming at a complete cluster census out to ever higher redshifts, e.g. eROSITA (Predehl et al. 2010; Merloni et al. 2012; Pillepich et al. 2012) and Athena (Nandra et al. 2013; Pointecouteau et al. 2013) in X-rays, Euclid (Laureijs et al. 2011; Amendola et al. 2013), DES and LSST in the optical/near-infrared, CCAT (Woody et al. 2012) and SKA at sub-mm and radio frequencies.

Careful X-ray studies of clusters at low and intermediate redshifts yield highly precise cluster masses, but assume hydrostatic equilibrium, and in most cases spherical symmetry (e.g. Croston et al. 2008; Ettori et al. 2013). Observational evidence and numerical modelling challenge these assumptions for all but the most relaxed systems (e.g. Mahdavi et al. 2008; Rasia et al. 2012; Limousin et al. 2013; Newman et al. 2013). While simulations find X-ray masses to only slightly underestimate the true mass of clusters that exhibit no indications of recent mergers and can be considered virialised, non-thermal pressure support 
can lead to a $>20 \%$ bias in unrelaxed clusters (Laganá et al. 2010; Rasia et al. 2012). Shi \& Komatsu (2014) modelled the pressure due to ICM turbulence analytically and found a $\sim 10 \%$ underestimate of cluster masses compared to the hydrostatic case.

Weak lensing (WL), in contrast, is subject to larger stochastic uncertainties, but can in principle yield unbiased masses, as no equilibrium assumptions are required. Details of the mass modelling however can introduce biases, in particular concerning projection effects, the source redshift distribution and the departures from an axisymmetric mass profile (Corless \& King 2009; Becker \& Kravtsov 2011; Bahé et al. 2012; Hoekstra et al. 2013). For individual clusters, stochastic uncertainties dominate the budget; however, larger cluster samples benefit from improved corrections for lensing systematics, driven by cosmic shear projects (e.g. Massey et al. 2013).

Most of the leverage on cosmology and structure formation from future cluster surveys will be due to clusters at higher $z$ than have been previously investigated. Hence, the average cluster masses and signal/noise ratios for all observables are going to be smaller. Even and especially for the deepest surveys, most objects will lie close to the detection limit. Thus the scaling of inexpensive proxies (e.g. X-ray luminosity $L_{\mathrm{X}}$ ) with total mass needs to be calibrated against representative cluster samples at low and high $z$. Weak lensing and SZ mass estimates are both good candidates as they exhibit independent systematics from $\mathrm{X}$-rays and a weaker $z$-dependence in their signal/noise ratios.

Theoretically, cluster scaling relations arise from their description as self-similar objects forming through gravitational collapse (Kaiser 1986), and deviations from simple scaling laws provide crucial insights into cluster physics. For the current state of scaling relation science, we point to the recent review by Giodini et al. (2013). As we are interested in the cluster population to be seen by upcoming surveys, we focus here on results obtained at high redshifts.

Self-similar modelling includes evolution of the scaling relation normalisations with the Hubble expansion, which is routinely measured (e.g. Reichert et al. 2011; Ettori 2013). Evolution effects beyond self-similarity, e.g. due to declining AGN feedback at low $z$, have been claimed and discussed (e.g. Pacaud et al. 2007; Short et al. 2010; Stanek et al. 2010; Maughan et al. 2012), but current observations are insufficient to constrain possible evolution in slopes (Giodini et al. 2013). Evidence for different scaling behaviour in groups and low-mass clusters was found by, e.g., Eckmiller et al. (2011), Stott et al. (2012), Bharadwaj et al. (2014).

Reichert et al. (2011) and Maughan et al. (2012) investigated X-ray scaling relationships including clusters at $z>1$, and both stressed the increasing influence of selection effects at higher $z$. Larger weak lensing samples of distant clusters are just in the process of being compiled (Jee et al. 2011; Foëx et al. 2012; Hoekstra et al. 2011a, 2012; Israel et al. 2012; von der Linden et al. 2014a; Postman et al. 2012). Thus most WL scaling studies are currently limited to $z \lesssim 0.6$, and also include nearby clusters (e.g. Hoekstra et al. 2012; Mahdavi et al. 2013, M13). The latter authors find projected WL masses follow the expected correlation with the SZ signal $Y_{\mathrm{SZ}}$, corroborating similar results for more local clusters by Marrone et al. (2009, 2012). Miyatake et al. (2013) performed a detailed WL analysis of a $z=0.81$ cluster discovered in the SZ using the Atacama Cosmology Telescope, and compared the resulting lensing mass against the Reese et al. (2012) $Y_{\mathrm{SZ}}-M$ scaling relation, in what they describe as a first step towards a high- $z$ SZ-WL scaling study.
By compiling Hubble Space Telescope data for 27 massive clusters at $0.83<z<1.46$, Jee et al. (2011) not only derive the relation between WL masses $M^{\mathrm{wl}}$ and ICM temperature $T_{\mathrm{X}}$, but also notice a good correspondence between WL and hydrostatic X-ray masses $M^{\text {hyd }}$. As they focus on directly testing cosmology with the most massive clusters, these authors however stop short of deriving the WL-X-ray scaling. Also using HST observations, Hoekstra et al. (2011a) investigated the WL mass scaling of the optical cluster richness (i.e. galaxy counts) and $L_{\mathrm{X}}$ of 25 moderate- $L_{\mathrm{X}}$ clusters at $0.3<z<0.6$, thus initiating the study of WL scaling relations off the top of the mass function.

Comparisons between weak lensing and X-ray masses for larger cluster samples were pioneered by Mahdavi et al. (2008) and Zhang et al. (2008), collecting evidence for the ratio of weak lensing to X-ray masses $M^{\mathrm{wl}} / M^{\text {hyd }}>1$, indicating non-thermal pressure. Zhang et al. (2010), analysing 12 clusters from the Local Cluster Substructure Survey (LoCuSS), find this ratio to depend on the radius. Likewise, a difference between relaxed and unrelaxed clusters is found (Zhang et al. 2010; Mahdavi et al. 2013). Rasia et al. (2012) show that the gap between X-ray and lensing masses is more pronounced in simulations than in observations, pointing to either an underestimate of the true mass also by WL masses (cf. Bahé et al. 2012) or to simulations overestimating the X-ray mass bias.

The current disagreement between the cosmological constraints derived from Planck primary cosmic microwave background (CMB) data with Wilkinson Microwave Anisotropy Probe data, supernova data, and cluster data (Planck Collaboration XX 2014) may well be alleviated by, e.g. sliding up a bit along the Planck degeneracy curve between the Hubble factor $H_{0}$ and the matter density parameter $\Omega_{\mathrm{m}}$. Nevertheless, as stronger cluster mass biases than currently favoured $(\sim 40 \%)$ have also been invoked as a possible explanation, it is very important to test the cluster mass calibration with independent methods out to high $z$, as we do in this work.

This article aims to test the agreement of the weak lensing and X-ray masses measured by Israel et al. (2012) for 8 relatively low-mass clusters at $z \gtrsim 0.4$ with scaling relations from the recent literature. The $400 \mathrm{~d} \mathrm{X}$-ray sample from which our clusters are drawn has been constructed to contain typical objects at intermediate redshifts, similar in mass and redshift to upcoming surveys. Hence, it does not include extremely massive low- $z$ clusters. We describe the observations and WL and X-ray mass measurements for the 8 clusters in Sect. 2, before presenting the central scaling relations in Sect. 3. Possible explanations for the steep slopes our scaling relations exhibit are discussed in Sect. 4, and we compare to literature results in Sect. 5, leading to the conclusions in Sect. 6. Throughout this article, $E(z)=H(z) / H_{0}=\sqrt{\Omega_{\mathrm{m}}(z+1)^{3}+\Omega_{\Lambda}}$ denotes the selfsimilar evolution factor (Hubble factor $H(z)$ normalised to its present-day value of $H_{0}=72 \mathrm{~km} \mathrm{~s}^{-1} \mathrm{Mpc}^{-1}$ ), computed for a flat universe with matter and dark energy densities of $\Omega_{\mathrm{m}}=0.3$ and $\Omega_{\Lambda}=0.7$ in units of the critical density.

\section{Observations and data analysis}

\subsection{The $400 d$ weak lensing survey}

This article builds on the weak lensing analysis for 8 clusters of galaxies (Israel et al. 2010, 2012, Paper I and Paper II hereafter) selected from the 400d X-ray selected sample of clusters (Burenin et al. 2007; Vikhlinin et al. 2009a, V09a). From the $\sim 400 \mathrm{deg}^{2}$ of all suitable ROSAT PSPC observations, 
Burenin et al. (2007) compiled a catalogue of serendipitously detected clusters, i.e. discarding the intentional targets of the ROSAT pointings. For a uniquely complete subsample of 36 X-ray luminous $\left(L_{\mathrm{X}} \gtrsim 10^{44} \mathrm{erg} / \mathrm{s}\right)$ high-redshift $(0.35 \leq z \leq$ 0.89) sources, V09a obtained deep Chandra data, weighing the clusters using three different mass proxies (Sect. 3.2). Starting from the cluster mass function computed by V09a, Vikhlinin et al. (2009b) went on to constrain cosmological parameters. For brevity, we will refer to the V09a high-z sample as the $400 \mathrm{~d}$ sample. The $400 \mathrm{~d}$ weak lensing survey follows up these clusters in weak lensing, determining independent WL masses with the ultimate goals of deriving the lensingbased mass function for the complete sample and to perform detailed consistency checks. Currently, we have determined WL masses for 8 clusters observed in four dedicated MMT/Megacam runs (see Papers I and II). Thus, our scaling relation studies are largely limited to this subset of clusters, covering the sky between $\alpha_{\mathrm{J} 2000}=13^{\mathrm{h}} 30^{\mathrm{m}}-24^{\mathrm{h}}$ with $\delta_{\mathrm{J} 2000}>10^{\circ}$ and $\alpha_{\mathrm{J} 2000}=0^{\mathrm{h}}-08^{\mathrm{h}} 30^{\mathrm{m}}$ with $\delta_{\mathrm{J} 2000}>0^{\circ}$.

\subsection{Weak lensing analysis}

We present only a brief description of the WL analysis in this paper; for more details see Paper II. Basic data reduction is performed using the THELI pipeline for multi-chip cameras (Erben et al. 2005; Schirmer 2013), adapted to MMT/Megacam. We employ the photometric calibration by Hildebrandt et al. (2006). Following Dietrich et al. (2007), regions of the THELI coadded images not suitable for WL shear measurements are masked. Shear is measured using an implementation of the "KSB+" algorithm (Kaiser et al. 1995; Erben et al. 2001), the "TS" pipeline (Heymans et al. 2006; Schrabback et al. 2007; Hartlap et al. 2009). Catalogues of lensed background galaxies are selected based on the available colour information. For clusters covered in three filters, we include galaxies based on their position in colour-colour-magnitude space (Paper II; see Klein et al., in prep., for a generalisation). For clusters covered only in one passband, we apply a magnitude cut. Where available, colour information also enables us to quantify and correct for the dilution by residual cluster members (Hoekstra 2007) in the shear catalogues. The mass normalisation of the WL signal is set by the mean lensing depth $\langle\beta\rangle$, defined as $\beta=D_{\mathrm{ds}} / D_{\mathrm{s}}$, the ratio of angular diameter distances between the deflector and the source, and between the observer and the source. The Ilbert et al. (2006) CFHTLS Deep fields photometric redshift catalogue serves as a proxy for estimating $\langle\beta\rangle$ and for calibrating the background selection.

The tangential ellipticity profiles given the ROSAT cluster centres are modelled by fitting the reduced shear profile (Bartelmann 1996; Wright \& Brainerd 2000) corresponding to the Navarro et al. (1996, 1997, NFW) density profile between $0.2 \mathrm{Mpc}$ and 5.0 Mpc projected radius. Input ellipticities are scaled according to the Hartlap et al. (2009) calibration factor and, where applicable, with the correction for dilution by cluster members. We consider the intrinsic source ellipticity measured from the data, accounting for its dependence on the shear (Schneider et al. 2000).

Lensing masses are inferred by evaluating a $\chi^{2}$ merit function on a grid in radius $r_{200}$ and concentration $c_{200}$. The latter is poorly constrained in the direct fit, so we marginalise over it assuming an empirical massconcentration relation. In addition to the direct fit approach, in Israel et al. (2012), we report masses using two different mass-concentration relations: Bullock et al. (2001, B01), and
Bhattacharya et al. (2013, B13) ${ }^{1}$. Finding the masses using B01 or B13 makes them less susceptible to variations in the model in Paper II, we explore their effect further in Sect. 4.1.

\subsection{Choice of the overdensity contrast}

Cluster scaling relations are usually given for the mass contained within a radius $r_{500}$, corresponding to an overdensity $\Delta=500$ compared to the critical density $\rho_{\mathrm{c}}$ of the Universe at the cluster redshift. This $\Delta$ is chosen because the best-constrained X-ray masses are found close to $r_{500}$, determined by the particle backgrounds of Chandra and XMM-Newton (cf. Okabe et al. 2010a). Currently, only Suzaku allows direct constraints upon X-ray masses at $r_{200}$ (see Reiprich et al. 2013, and references therein). In order to compare to the results from the Vikhlinin et al. (2009a) Chandra analysis, we compute our $\Delta=500 \mathrm{WL}$ masses from our $\Delta=200$ masses, assuming the fitted NFW profiles given by $\left(r_{200}, c_{200}\right)$ to be correct. Independent of $\Delta>1$, the cumulative mass of a NFW halo, described by $r_{\Delta}$ and $c_{\Delta}$, out to a test radius $r$ is given by:

$$
\begin{aligned}
M_{\mathrm{NFW}}(r) & =\Delta \rho_{\mathrm{c}} \frac{4 \pi}{3} r_{\Delta}^{3} \times \frac{\ln \left(1+c_{\Delta} r / r_{\Delta}\right)-\frac{c_{\Delta} r / r_{\Delta}}{1+c_{\Delta} r / r_{\Delta}}}{\ln \left(1+c_{\Delta}\right)-c_{\Delta} /\left(1+c_{\Delta}\right)} \\
& =M_{\Delta}\left(r_{\Delta}\right) \times \Xi\left(r ; r_{\Delta}, c_{\Delta}\right),
\end{aligned}
$$

separating into the mass $M_{\Delta}$ and a function we call $\Xi\left(r ; r_{\Delta}, c_{\Delta}\right)$. Equating Eq. (1) with $r=r_{500}$ for $\Delta=200$ and $\Delta^{\prime}=500$, we arrive at this implicit equation for $r_{500}$, which we solve numerically:

$r_{500}=r_{200}\left(\frac{2}{5} \Xi\left(r_{500}, r_{200}, c_{200}\right)\right)^{1 / 3}$.

\subsection{X-ray analysis}

Under the strong assumptions that the ICM is in hydrostatic equilibrium and follows a spherically symmetric mass distribution, the cluster mass within a radius $r$ can be calculated as (see e.g. Sarazin 1988):

$M^{\text {hyd }}(r)=\frac{-k_{\mathrm{B}} T_{\mathrm{X}}(r) r}{\mu m_{\mathrm{p}} G}\left(\frac{\mathrm{d} \ln \rho_{\mathrm{g}}(r)}{\mathrm{d} \ln r}+\frac{\mathrm{d} \ln T_{\mathrm{X}}(r)}{\mathrm{d} \ln r}\right)$

from the ICM density and temperature profiles $\rho_{\mathrm{g}}(r)$ and $T_{\mathrm{X}}(r)$, where $G$ is the gravitational constant, $m_{\mathrm{p}}$ is the proton mass, and $\mu=0.5954$ the mean molecular mass of the ICM. The ICM density is modelled by fitting the observed Chandra surface brightness profile, assuming a primordial He abundance and an ICM metallicity of $0.2 Z_{\odot}$, such that $\rho_{\mathrm{g}}(r)=1.274 m_{\mathrm{p}} n(r)$. We use a Vikhlinin et al. (2006) particle density profile with $n(r)=$ $\sqrt{n_{\mathrm{p}}(r) n_{\mathrm{e}}(r)}$. Extending the widely-used $\beta$-profile (Cavaliere \& Fusco-Femiano 1978), it allows for prominent cluster cores as well as steeper surface brightness profiles in the cluster outskirts to be modelled by additional terms. Regarding the systematic differences Rozo et al. (2014b) find between X-ray mass algorithms, we point out that we employ the V09a profiles directly rather than re-deriving them from their parameters.

The relatively low signal/noise in the Chandra data renders the determination of individual temperature profiles difficult. Rather, we fit a global $T_{\mathrm{X}}$ (Table 1; V09a) and assume the empirical average temperature profile $T_{\mathrm{X}}(r)=T_{\mathrm{X}}\left(1.19-0.84 r / r_{200}\right)$ Reiprich et al. (2013) derive from compiling all available $S u z a k u$

\footnotetext{
1 Actually, we use the slightly different relation as given in Version 1, referred to in Paper II as "B12": arxiv .org/abs/1112 . 5479v1
} 
Table 1. Measured properties of the 400d MMT cluster sample.

\begin{tabular}{|c|c|c|c|c|c|c|c|c|}
\hline & $\begin{array}{c}\text { CL } 0030 \\
+2618 \\
\end{array}$ & $\begin{array}{c}\text { CL } 0159 \\
+0030 \\
\end{array}$ & $\begin{array}{c}\text { CL } 0230 \\
+1836 \\
\end{array}$ & $\begin{array}{c}\text { CL } 0809 \\
+2811 \\
\end{array}$ & $\begin{array}{c}\text { CL } 1357 \\
+6232 \\
\end{array}$ & $\begin{array}{c}\text { CL } 1416 \\
+4446 \\
\end{array}$ & $\begin{array}{c}\text { CL } 1641 \\
+4001 \\
\end{array}$ & $\begin{array}{c}\text { CL } 1701 \\
+6414 \\
\end{array}$ \\
\hline Redshift $z$ & 0.50 & 0.39 & 0.80 & 0.40 & 0.53 & 0.40 & 0.46 & 0.45 \\
\hline$k_{\mathrm{B}} T_{\mathrm{X}}$ from V09a [keV] & $5.63 \pm 1.13$ & $4.25 \pm 0.96$ & $5.50 \pm 1.02$ & $4.17 \pm 0.73$ & $4.60 \pm 0.69$ & $3.26 \pm 0.46$ & $3.31 \pm 0.62$ & $4.36 \pm 0.46$ \\
\hline$M_{500}^{\mathrm{Y}}$ from V09a & $3.43 \pm 0.41$ & $2.51 \pm 0.37$ & $3.46 \pm 0.46$ & $3.69 \pm 0.42$ & $2.96 \pm 0.29$ & $2.52 \pm 0.24$ & $1.70 \pm 0.20$ & $3.28 \pm 0.24$ \\
\hline$M_{500}^{\mathrm{T}}$ from V09a & $4.41 \pm 1.33$ & $2.67 \pm 0.90$ & $3.57 \pm 0.99$ & $2.96 \pm 0.78$ & $2.78 \pm 0.62$ & $1.76 \pm 0.37$ & $1.73 \pm 0.49$ & $2.66 \pm 0.42$ \\
\hline$M_{500}^{\mathrm{G}}$ from $\mathrm{V} 09 \mathrm{a}$ & $2.04 \pm 0.19$ & $1.92 \pm 0.22$ & $2.70 \pm 0.27$ & $3.98 \pm 0.35$ & $2.40 \pm 0.18$ & $3.10 \pm 0.24$ & $1.34 \pm 0.13$ & $3.20 \pm 0.20$ \\
\hline$r_{200}^{\mathrm{wl}}\left(c_{\mathrm{fit}}\right)[\mathrm{kpc}]$ from Paper II & $1520_{-160}^{+140}$ & $1370_{-220}^{+180}$ & $1540_{-320}^{+280}$ & $1750_{-280}^{+230}$ & $1110_{-250}^{+210}$ & $980_{-180}^{+150}$ & $1060_{-260}^{+300}$ & $940_{-290}^{+320}$ \\
\hline$r_{200}^{\mathrm{wl}}\left(c_{\mathrm{B} 13}\right)[\mathrm{kpc}]$ from Paper II & $1445_{-151}^{+130}$ & $1312_{-275}^{+222}$ & $1514_{-317}^{+270}$ & $1714_{-221}^{+192}$ & $1057_{-236}^{+202}$ & $977_{-183}^{+150}$ & $1014_{-126}^{+260}$ & $1014_{-201}^{+160}$ \\
\hline$r_{200}^{\mathrm{wl}}\left(c_{\mathrm{fit}}\right)$, no dilu. corr. [kpc] & $1430_{-150}^{+140}$ & $1320_{-200}^{+170}$ & $1470_{-310}^{+280}$ & $1660_{-270}^{+220}$ & - & - & - & - \\
\hline$r_{200}^{\mathrm{wl}}\left(c_{\mathrm{B} 13}\right)$, no dilu. corr. [kpc] & $1349_{-136}^{+123}$ & $1265_{-256}^{+214}$ & $1432_{-305}^{+258}$ & $1629_{-204}^{+182}$ & - & - & - & - \\
\hline$r_{500}^{\mathrm{wl}}\left(c_{\mathrm{fit}}\right)[\mathrm{kpc}]$ & $914_{-96}^{+84}$ & $959_{-154}^{+126}$ & $974_{-203}^{+177}$ & $994_{-159}^{+131}$ & $674_{-152}^{+127}$ & $651_{-120}^{+100}$ & $440_{-108}^{+125}$ & $404_{-125}^{+137}$ \\
\hline$r_{500}^{\mathrm{wl}}\left(c_{\mathrm{B} 13}\right)[\mathrm{kpc}]$ & $933_{-96}^{+84}$ & $848_{-175}^{+144}$ & $959_{-203}^{+171}$ & $1108_{-142}^{+129}$ & $685_{-155}^{+129}$ & $637_{-117}^{+104}$ & $655_{-182}^{+108}$ & $655_{-130}^{+110}$ \\
\hline$r_{500}^{\mathrm{wl}}\left(c_{\mathrm{fit}}\right)$, no dilu. corr. $[\mathrm{kpc}]$ & $850_{-89}^{+83}$ & $924_{-140}^{+119}$ & $919_{-194}^{+175}$ & $923_{-150}^{+122}$ & - & - & - & - \\
\hline$r_{500}^{\mathrm{wl}}\left(c_{\mathrm{B} 13}\right)$, no dilu. corr. $[\mathrm{kpc}]$ & $870_{-90}^{+87}$ & $816_{-168}^{+136}$ & $909_{-191}^{+165}$ & $1051_{-129}^{+116}$ & - & - & - & - \\
\hline$r_{500}^{\mathrm{Y}}[\mathrm{kpc}]$ & $873 \pm 85$ & $821 \pm 109$ & $777 \pm 75$ & $930 \pm 84$ & $821 \pm 92$ & $819 \pm 108$ & $702 \pm 138$ & $877 \pm 89$ \\
\hline$r_{500}^{\mathrm{T}}[\mathrm{kpc}]$ & $949 \pm 72$ & $838 \pm 105$ & $785 \pm 73$ & $864 \pm 97$ & $804 \pm 96$ & $727 \pm 138$ & $706 \pm 136$ & $818 \pm 102$ \\
\hline$r_{500}^{\mathrm{G}}[\mathrm{kpc}]$ & $734 \pm 120$ & $751 \pm 130$ & $715 \pm 88$ & $954 \pm 80$ & $766 \pm 106$ & $877 \pm 94$ & $648 \pm 161$ & $870 \pm 91$ \\
\hline$M_{500}^{\mathrm{wl}}\left(r_{500}^{\mathrm{wl}}\right)$, using $c_{\mathrm{fit}}$ & $3.94_{-1.12}^{+1.19}$ & $4.00_{-1.63}^{+1.79}$ & $6.82_{-3.44}^{+4.43}$ & $4.51_{-1.84}^{+2.03}$ & $1.64_{-0.88}^{+1.11}$ & $1.27_{-0.58}^{+0.68}$ & $0.42_{-0.24}^{+0.47}$ & $0.32_{-0.22}^{+0.45}$ \\
\hline$M_{500}^{\mathrm{wl}}\left(r_{500}^{\mathrm{wl}}\right)$, using $c_{\mathrm{B} 13}$ & $4.19_{-1.16}^{+1.23}$ & $2.77_{-1.38}^{+1.64}$ & $6.51_{-3.32}^{+4.14}$ & $6.24_{-2.11}^{+2.24}$ & $1.72_{-0.92}^{+1.16}$ & $1.19_{-0.54}^{+0.68}$ & $1.38_{-0.86}^{+1.05}$ & $1.37_{-0.66}^{+0.81}$ \\
\hline$M_{500}^{\mathrm{wl}}\left(r_{500}^{\mathrm{wl}}\right)$, using $c_{\text {fit }}$, no dilu. corr. & $3.17_{-0.89}^{+1.02}$ & $3.58_{-1.39}^{+1.50}$ & $5.73_{-2.92}^{+3.93}$ & $3.61_{-1.49}^{+1.63}$ & - & - & - & - \\
\hline$M_{500}^{\mathrm{wl}}\left(r_{500}^{\mathrm{wl}}\right)$, using $c_{\mathrm{B} 13}$, no dilu. corr. & $3.40_{-0.95}^{+0.98}$ & $2.46_{-1.23}^{+1.45}$ & $5.54_{-2.81}^{+3.60}$ & $5.33_{-1.73}^{+1.97}$ & - & - & - & - \\
\hline$M_{500}^{\mathrm{wl}}\left(r_{500}^{\mathrm{Y}}\right)$, using $c_{\mathrm{B} 13}$ & $3.91_{-0.83}^{+0.78}$ & $2.68_{-1.12}^{+0.93}$ & $5.15_{-2.07}^{+1.83}$ & $5.15_{-1.34}^{+1.25}$ & $2.04_{-0.96}^{+0.81}$ & $1.49_{-0.59}^{+0.52}$ & $1.48_{-0.85}^{+0.64}$ & $1.78_{-0.76}^{+0.64}$ \\
\hline$M_{500}^{\mathrm{wl}}\left(r_{500}^{\mathrm{T}}\right)$, using $c_{\mathrm{B} 13}$ & $4.26_{-0.91}^{+0.83}$ & $2.73_{-1.15}^{+0.95}$ & $5.21_{-2.10}^{+1.85}$ & $4.76_{-1.24}^{+1.17}$ & $2.00_{-0.94}^{+0.79}$ & $1.34_{-0.52}^{+0.47}$ & $1.48_{-0.85}^{+0.64}$ & $1.68_{-0.71}^{+0.60}$ \\
\hline$M_{500}^{\mathrm{wl}}\left(r_{500}^{\mathrm{G}}\right)$, using $c_{\mathrm{B} 13}$ & $3.26_{-0.70}^{+0.69}$ & $2.45_{-1.02}^{+0.85}$ & $4.68_{-1.86}^{+1.66}$ & $5.29_{-1.38}^{+1.28}$ & $1.91_{-0.89}^{+0.75}$ & $1.58_{-0.63}^{+0.56}$ & $1.37_{-0.78}^{+0.59}$ & $1.77_{-0.75}^{+0.64}$ \\
\hline$M_{500}^{\mathrm{hyd}}\left(r_{500}^{\mathrm{wl}}\right)$, using $c_{\mathrm{fit}}$ & $3.46_{-1.01}^{+1.10}$ & $2.78_{-1.00}^{+1.11}$ & $5.13_{-2.37}^{+2.95}$ & $3.66_{-1.26}^{+1.36}$ & $2.20_{-0.85}^{+0.92}$ & $1.31_{-0.44}^{+0.51}$ & $1.17_{-0.51}^{+0.70}$ & $1.02_{-0.45}^{+0.60}$ \\
\hline$M_{500}^{\text {hyd }}\left(r_{500}^{\text {wl }}\right)$, using $c_{\mathrm{B} 13}$ & $3.47_{-1.00}^{+1.10}$ & $2.50_{-1.101}^{+1.12}$ & $4.99_{-2.33}^{+2.81}$ & $3.99_{-1.22}^{+1.20}$ & $2.20_{-0.95}^{+0.09}$ & $1.28_{-0.43}^{+0.0 .44}$ & $1.65_{-0.72}^{-0.01}$ & $1.70_{-0.52}^{+0.43}$ \\
\hline$M_{500}^{\text {hyd }}\left(r_{500}^{\text {wl }}\right)$, using $c_{\text {fit }}$, no dilu. corr. & $3.21_{-0.94}^{+1.05}$ & $2.68_{-0.94}^{+1.06}$ & $4.66_{-2.18}^{+2.81}$ & $3.35_{-1.18}^{+1.27}$ & - & - & - & - \\
\hline$M_{500}^{\mathrm{hyd}}\left(r_{500}^{\mathrm{wl}}\right)$, using $c_{\mathrm{B} 13}$, no dilu. corr. & $3.22_{-0.94}^{+1.02}$ & $2.40_{-0.97}^{+1.08}$ & $4.54_{-2.12}^{+2.16}$ & $3.75_{-1.13}^{+1.10}$ & - & - & - & - \\
\hline
\end{tabular}

Notes. We quote the properties adopted from V09a and state the overdensity radii and corresponding cluster masses used for Figs. 1 and 2. All masses are in units of $10^{14} M_{\odot}$, without applying the $E(z)$ factor. We state only stochastic uncertainties, i.e. do not include systematics. The proxybased masses $M_{500}^{\mathrm{Y}, \mathrm{T}, \mathrm{G}}$ from V09a in the first part of this table are defined in Sect. 2.5, as well as the $r_{500}^{\mathrm{Y}, \mathrm{T}, \mathrm{G}}$ quoted in the second part. The third and fourth part of the table show the weak lensing $\left(M^{\mathrm{wl}}\right)$ and hydrostatic $\left(M^{\text {hyd }}\right)$ for different cases, respectively. By $c_{\mathrm{fit}}$ and $c_{\mathrm{B} 13}$ we denote the choices for the NFW concentration parameter explained in Sect. 2.2. We refer to Sect. 4.3 for the introduction of the "no dilu. corr." case (only differing from the default for the first four clusters). See Table A.1 for further properties.

temperature profiles (barring only the two most exceptional clusters). For $r_{200}$, we use the WL results from Paper $\mathrm{II}^{2}$.

Equation (4) provides us with a cumulative mass profile. We evaluate this profile at some $r_{\text {test }}$, e.g. from WL, and propagate the uncertainty in $r_{\text {test }}$, together with the uncertainty in $T_{\mathrm{X}}$.

Hydrostatic equilibrium and sphericity are known to be problematic assumptions for many clusters. Nonetheless, hydrostatic masses are commonly used in the literature in comparisons to WL masses. Our goal is to study if and how biases due to deviations from the above-mentioned assumptions show up.

\subsection{Mass estimates}

Table 1 comprises the key results on radii $r_{500}$ and the corresponding mass estimates. By $M^{\mathcal{P}}\left(r^{Q}\right)$, we denote a mass measured from data on proxy $\mathcal{P}$ within a radius defined by proxy $Q$.

\footnotetext{
2 For the scaling relations within WL-derived radii, we choose the respective $c_{\mathrm{NFW}}$. Otherwise, we use $c_{\mathrm{B} 13}$ as a default.
}

We use five mass estimates: $\mathcal{P}, \mathcal{Q} \in\{$ wl, hyd, Y, T, G $\}$. The first two are the weak lensing (wl) and hydrostatic X-ray masses (hyd), as introduced in Sects. 2.2 and 2.4. Having analysed deep Chandra observations they acquired, Vikhlinin et al. (2009a) present three further mass estimates for all 36 clusters in the complete sample. Based on the proxies $T_{\mathrm{X}}$, the ICM mass $M_{\text {gas }}$, and $Y_{\mathrm{X}}=T_{\mathrm{X}} M_{\text {gas }}$, mass estimates $M^{\mathrm{T}}, M^{\mathrm{G}}$, and $M^{\mathrm{Y}}$ are quoted in Table 2 of V09a. We point out that V09a obtain these estimates by calibrating the mass scaling relations for respective proxy on local clusters (see their Table 3). V09a further provide a detailed account of the relevant systematic sources of uncertainty.

The radii $r_{500}^{\mathcal{P}}=\left(3 M_{500}^{\mathcal{P}} /\left(2000 \pi \rho_{\mathrm{c}}\right)\right)^{1 / 3}$ listed in Table 1 are obtained from $M_{500}^{\mathcal{P}}, \mathcal{P} \in\{\mathrm{Y}, \mathrm{T}, \mathrm{G}\}$. Using Eqs. (1) and (4), we then derive the WL and hydrostatic masses, respectively, within these radii. We emphasise that all WL mass uncertainties quoted in Table 1 are purely statistical and do not include any of the systematics discussed in Paper II. 


\subsection{Fitting algorithm for scaling relations}

The problem of selecting the best linear representation $y=$ $A+B x$ for a sample of (astronomical) observations of two quantities $\left\{x_{i}\right\}$ and $\left\{y_{i}\right\}$ can be surprisingly complex. A plethora of algorithms and literature cope with the different assumptions about measurement uncertainties one can or has to make (e.g. Press et al. 1992; Akritas \& Bershady 1996; Tremaine et al. 2002; Kelly 2007; Hogg et al. 2010; Williams et al. 2010; Andreon \& Hurn 2012; Feigelson \& Babu 2012). The challenges observational astronomers have to tackle when trying to reconcile the prerequisites of statistical estimators with the realities of astrophysical data are manifold, including heteroscedastic uncertainties (i.e. depending non-trivially on the data themselves), intrinsic scatter, poor knowledge of systematics, poor sample statistics, "outlier" points, and non-Gaussian probability distributions. Tailored to the problem of galaxy cluster scaling relations, Maughan (2014) proposed a "self-consistent" modelling approach based on the fundamental observables. A full account of these different effects exceeds the scope of this article. We choose the relatively simple fitexy algorithm (Press et al. 1992), minimising the estimator

$\chi_{\mathrm{P} 92}^{2}=\sum_{i=1}^{N} \frac{\left(y_{i}-A-B x_{i}\right)^{2}}{\sigma_{y, i}^{2}+B^{2} \sigma_{x, i}^{2}}$,

which allows the uncertainties $\sigma_{x, i}$ and $\sigma_{y, i}$ to vary for different data points $x_{i}$ and $y_{i}$, but assumes them to be drawn from a Gaussian distribution. To accommodate intrinsic scatter, $\sigma_{y, i}^{2}$ in Eq. (5) can be replaced by $\sigma_{i}^{2}=\sigma_{y, i}^{2}+\sigma_{\text {int }}^{2}$ (e.g. Weiner et al. 2006; Andreon \& Hurn 2012). We test for intrinsic scatter using mpfitexy (Markwardt 2009; Williams et al. 2010), but in most cases, due to the small $\chi^{2}$ values, find the respective parameter not invoked. Thus we decide against this additional complexity. A strength of Eq. (5) is its invariance under changing $x$ and $y$ (e.g. Tremaine et al. 2002); i.e., we do not assume either to be "the independent variable".

Rather than propagating the (unknown) distribution functions in the mass uncertainties ${ }^{3}$, we approximate $1 \sigma$ Gaussian uncertainties in decadic log-space, applying the symmetrisation:

$\sigma_{\left(\log \xi_{i}\right)}=\log (\mathrm{e})\left(\xi_{i}^{+}-\xi_{i}^{-}\right) /\left(2 \xi_{i}\right)=\log (\mathrm{e})\left(\sigma_{\xi, i}^{+}+\sigma_{\xi, i}^{-}\right) /\left(2 \xi_{i}\right)$,

where $\xi_{i}^{+}=\xi_{i}+\sigma_{\xi, i}^{+}$and $\xi_{i}^{-}=\xi_{i}-\sigma_{\xi, i}^{-}$are the upper and lower limits of the $1 \sigma$ interval (in linear space) for the datum $\xi_{i}$, given the uncertainties $\sigma_{\xi, i}^{ \pm}$. All our calculations and plots use $\left\{x_{i}\right\}:=\left\{\log \xi_{i}\right\}$ and $\left\{\sigma_{x, i}\right\}:=\left\{\sigma_{\left(\log \xi_{i}\right)}\right\}$, with $\log \equiv \log _{10}$.

\section{Results}

\subsection{Weak lensing and hydrostatic masses}

The first and single most important observation is that hydrostatic masses $M_{500}^{\text {hyd }}\left(r_{500}^{\mathrm{wl}}\right)$, i.e. evaluated at $r_{500}$ as found from weak lensing, and weak lensing masses $M_{500}^{\mathrm{wl}}\left(r_{500}^{\mathrm{wl}}\right)$ roughly agree with each other (Table 1). Our second key observation is the very tight scaling behaviour between $M_{500}^{\text {hyd }}$ and $M_{500}^{\text {wl }}$, as Fig. 1 shows. In all cases presented in Fig. 1, and most of the ones we tested, all data points are consistent with the best-fit relation. Consequently, the fits return small values of $\chi_{\text {red }}^{2}<1$ (see

\footnotetext{
3 A natural feature in complex measurements like this, asymmetric uncertainties in $M_{200}^{\mathrm{wl}}$ arise from the grid approach to $\chi^{2}$ minimisation in Paper II (cf. Fig. 2 therein).
}
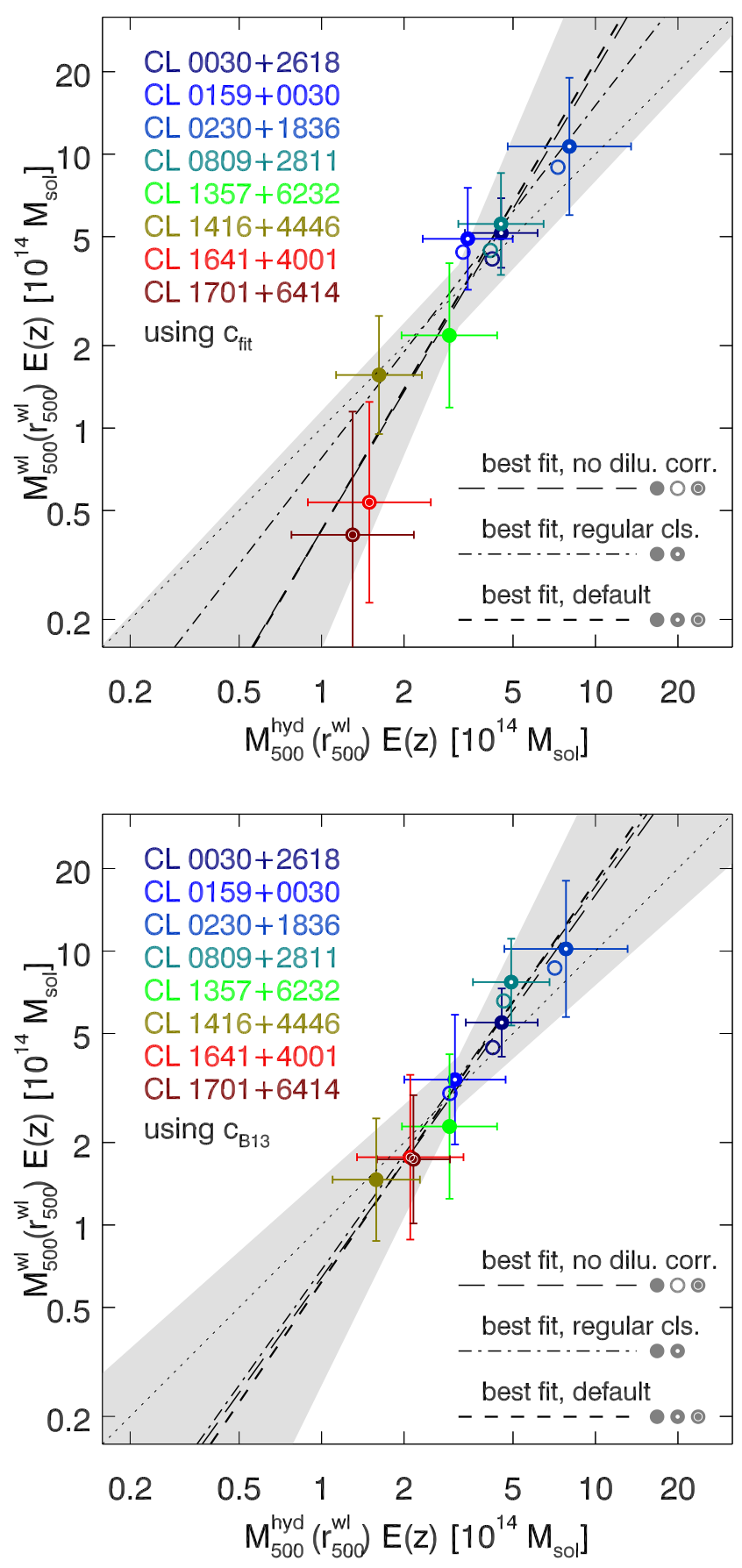

Fig. 1. Scaling of weak lensing masses $M_{500}^{\mathrm{wl}}\left(r_{500}^{\mathrm{wl}}\right)$ with hydrostatic masses $M_{500}^{\mathrm{hyd}}\left(r_{500}^{\mathrm{wl}}\right)$. The upper (lower) panel is for $c_{\mathrm{fit}}\left(c_{\mathrm{B} 13}\right)$. Both show best fits for three cases: the default (filled, thick ring, dotted ring symbols; thick dashed line), regular shear profile clusters only (filled and thick ring symbols; dash-dotted line; Sect. 4.1), and without correction for dilution by cluster members (filled, thin ring, dotted ring symbols; long dashed line; Sect. 4.3). The dotted line shows equality of the two masses, $M_{500}^{\mathrm{wl}}=M_{500}^{\text {hyd }}$. Shaded regions indicate the uncertainty range of the default best-fit. Some error bars were omitted for sake of clarity.

Table 2). Bearing in mind that we only use stochastic uncertainties, this points to some intrinsic correlation of the WL and hydrostatic masses. We will discuss this point in Sect. 4.2.

Finally, we find the slope of the $M_{500}^{\mathrm{wl}}\left(r_{500}^{\mathrm{wl}}\right)-M_{500}^{\mathrm{hyd}}\left(r_{500}^{\mathrm{wl}}\right)$ relation (dashed lines in Fig. 1) to be steeper than unity (dotted line): using the "default model", i.e. the analysis described in Sect. 2, a fitexy fit yields $1.71 \pm 0.64$ for the " $c_{\text {fit }}$ " case (concentration 
Table 2. Measurements of the X-ray - WL mass bias.

\begin{tabular}{lcccccccc}
\hline \hline Scaling relation & Model & $c_{\mathrm{NFW}}$ & Slope $B$ & Intercept $A$ & $b_{\mathrm{MC}}$ from Monte Carlo & \multicolumn{2}{c}{$b=\langle\log \xi-\log \eta\rangle \chi_{\text {red,M-M }}^{2}$ Section } \\
\hline$M_{500}^{\mathrm{wl}}\left(r_{500}^{\mathrm{wl}}\right)-M_{500}^{\mathrm{hyd}}\left(r_{500}^{\mathrm{wl}}\right)$ & default & $c_{\text {fit }}$ & $-0.51_{-0.21}^{+0.20}$ & $0.00_{-0.08}^{+0.07}$ & $0.08_{-0.13}^{+0.14}\left(0.27_{-0.20}^{+0.21} ;-0.10_{-0.15}^{+0.16}\right)$ & $0.08 \pm 0.09$ & 0.58 & 3.1 \\
& default & $c_{\mathrm{B} 13}$ & $-0.47_{-0.25}^{+0.26}$ & $-0.02_{-0.08}^{+0.07}$ & $0.00_{-0.13}^{+0.14}\left(0.10_{-0.18}^{+0.23} ;-0.10_{-0.15}^{+0.17}\right)$ & $-0.02 \pm 0.04$ & 0.52 & 3.1 \\
& no dilu. corr. & $c_{\text {fit }}$ & $-0.53 \pm 0.23$ & $0.01 \pm 0.08$ & $0.11_{-0.13}^{+0.14}\left(0.27_{-0.20}^{+0.21} ;-0.06_{-0.15}^{+0.16}\right)$ & $0.11 \pm 0.08$ & 0.57 & 4.3 \\
& no dilu. corr. & $c_{\mathrm{B} 13}$ & $-0.49 \pm 0.29$ & $-0.01_{-0.08}^{+0.07}$ & $0.02_{-0.13}^{+0.14}\left(0.10_{-0.18}^{+0.22} ;-0.06_{-0.15}^{+0.17}\right)$ & $0.00 \pm 0.03$ & 0.51 & 4.3 \\
\hline$M^{\mathrm{wl}}\left(r_{\text {fix }}\right)-M^{\mathrm{hyd}}\left(r_{\mathrm{fix}}\right)$ & $r_{\text {fix }}=600 \mathrm{kpc}$ & $c_{\mathrm{B} 13}$ & $-0.68_{-0.21}^{+0.19}$ & $-0.11 \pm 0.05$ & $0.01_{-0.07}^{+0.10}\left(0.12_{-0.10}^{+0.16} ;-0.11_{-0.08}^{+0.10}\right)$ & $-0.02 \pm 0.04$ & 0.82 & 4.2 \\
& $r_{\text {fix }}=800 \mathrm{kpc}$ & $c_{\mathrm{B} 13}$ & $-0.58_{-0.21}^{+0.19}$ & $-0.02 \pm 0.04$ & $0.02_{-0.07}^{+0.10}\left(0.12_{-0.11}^{+0.18} ;-0.09_{-0.08}^{+0.10}\right)$ & $-0.02 \pm 0.04$ & 0.72 & 4.2 \\
& $r_{\text {fix }}=1000 \mathrm{kpc}$ & $c_{\mathrm{B} 13}$ & $-0.52_{-0.21}^{+0.19}$ & $0.01 \pm 0.05$ & $0.01_{-0.08}^{+0.11}\left(0.10_{-0.11}^{+0.20} ;-0.09_{-0.09}^{+0.11}\right)$ & $-0.03 \pm 0.03$ & 0.69 & 4.2 \\
\hline$M_{500}^{\mathrm{wl}}\left(r_{500}^{\mathrm{Y}}\right)-M_{500}^{\mathrm{Y}}\left(r_{500}^{\mathrm{Y}}\right)$ & default & $c_{\mathrm{B} 13}$ & $-0.75_{-0.13}^{+0.12}$ & $0.07 \pm 0.03$ & $0.08_{-0.07}^{+0.10}\left(0.23_{-0.11}^{+0.18} ;-0.08_{-0.07}^{+0.10}\right)$ & $0.04 \pm 0.06$ & 1.21 & 3.2 \\
$M_{500}^{\mathrm{wl}}\left(r_{500}^{\mathrm{T}}\right)-M_{500}^{\mathrm{T}}\left(r_{500}^{\mathrm{T}}\right)$ & default & $c_{\mathrm{B} 13}$ & $-0.63 \pm 0.23$ & $0.04 \pm 0.06$ & $0.05_{-0.08}^{+0.11}\left(0.17_{-0.12}^{+0.11} ;-0.08_{-0.10}^{+0.11}\right)$ & $0.02 \pm 0.05$ & 0.88 & 3.2 \\
$M_{500}^{\mathrm{wl}}\left(r_{500}^{\mathrm{G}}\right)-M_{500}^{\mathrm{G}}\left(r_{500}^{\mathrm{G}}\right)$ & default & $c_{\mathrm{B} 13}$ & $-0.89_{-0.31}^{+0.18}$ & $0.01_{-0.04}^{+0.03}$ & $0.04_{-0.07}^{+0.10}\left(0.21_{-0.10}^{+0.11} ;-0.15_{-0.07}^{+0.09}\right)$ & $0.00 \pm 0.07$ & 2.11 & 3.2 \\
\hline
\end{tabular}

Notes. We estimate a possible bias between masses $\xi$ and $\eta$ by three estimators: First, we fit to $(\log \xi-\log \eta)$ as a function of $\eta$, yielding an intercept $A$ at pivot $\log \left(M_{\text {piv }} / M_{\odot}\right)=14.5$ and slope $B$ from the Monte Carlo/jackknife analysis. Second, we compute the logarithmic bias $b_{\mathrm{MC}}=\langle\log \xi-\log \eta\rangle_{\mathrm{MC}}$, averaged over the same realisations. Uncertainties for the MC results are given by $1 \sigma$ ensemble dispersions. In parentheses next to $b_{\mathrm{MC}}$, we show its value for the low- $M^{\mathrm{wl}}$ and high- $M^{\mathrm{wl}}$ clusters. Third, we quote the logarithmic bias $b=\langle\log \xi-\log \eta\rangle$ obtained directly from the input masses, along with its standard error. Finally, we give the $\chi_{\text {red }}^{2}$ for the mass-mass scaling, obtained from the MC method. The "default" model denotes WL and hydrostatic masses as described in Sect. 2.
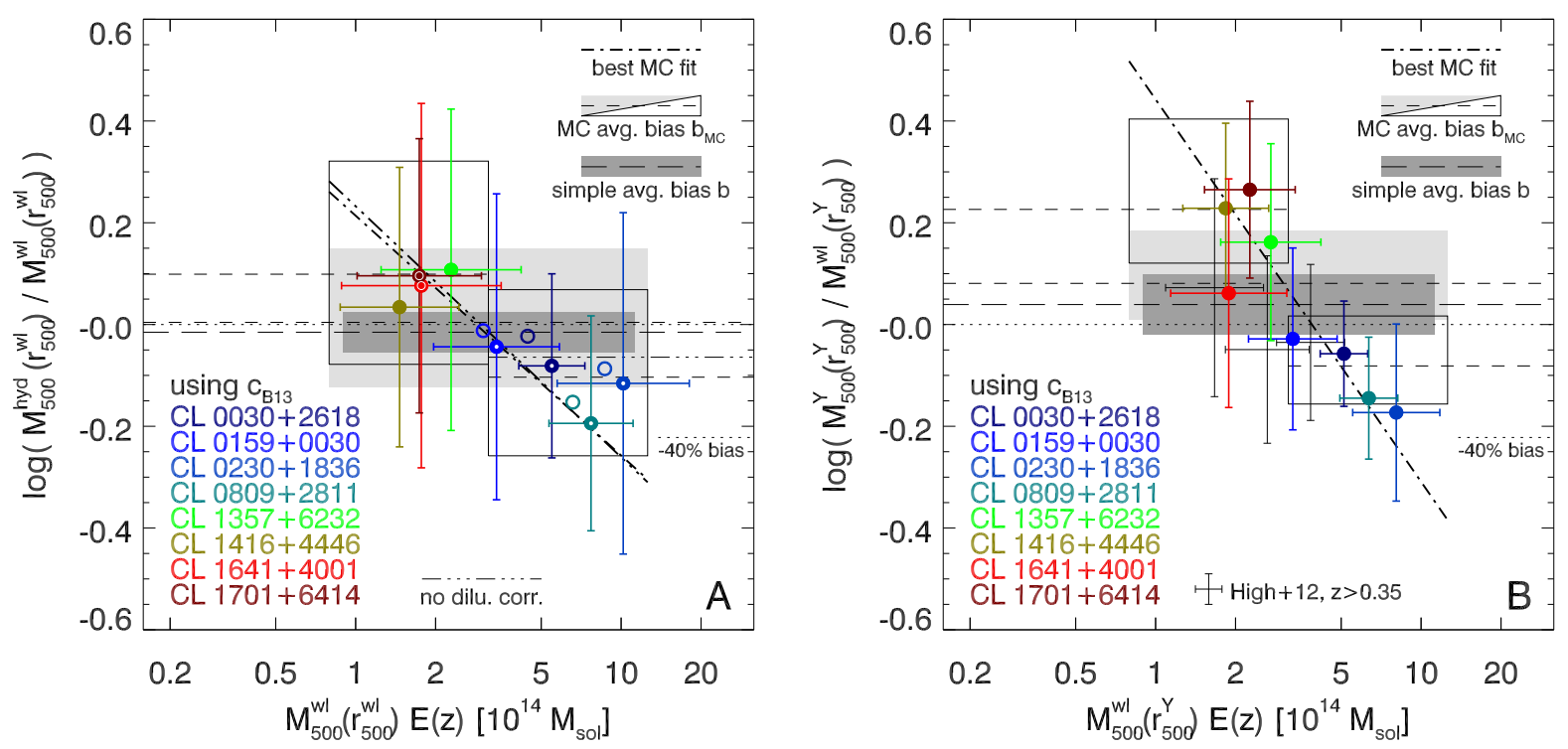

Fig. 2. Ratios between X-ray and WL masses as a function of WL mass. Panel A) shows $\log \left(M^{\mathrm{hyd}} / M^{\mathrm{wl}}\right)$ within $\left.r_{500}^{\mathrm{wl}}, \operatorname{panel} \mathbf{B}\right) \operatorname{shows} \log \left(M^{\mathrm{Y}} / M^{\mathrm{wl}}\right)$ within $r_{500}^{\mathrm{Y}}$. WL masses assume the B13 $c-M$ relation. We show three tests for a mass bias: the overall average $\operatorname{logarithmic}$ bias $b=\left\langle\log M^{\mathrm{X}}-\right.$ $\left.\log M^{\mathrm{wl}}\right\rangle$ is denoted by a long-dashed line, and its standard error by a dark grey shading. Short-dashed lines and light grey shading denote the same quantity, but obtained from averaging over Monte Carlo realisations including the jackknife test. We also show this $b_{\mathrm{MC}}$ for the low- $M^{\mathrm{wl}}$ and high- $M^{\mathrm{wl}}$ clusters separately, with the $1 \sigma$ uncertainties presented as boxes, for sake of clarity. As a visual aid, a dot-dashed line depicts the Monte Carlo/jackknife best-fit of $\log \left(M^{\mathrm{X}} / M^{\mathrm{wl}}\right)$ as a function of $M^{\mathrm{wl}}$. In addition, panel A) also contains this best-fit line (triple-dot-dashed) for the case without correction for cluster member dilution; the corresponding data points follow the Fig. 1 scheme. Indicated by uncertainty bars, panel B) also presents three high- $z$ clusters from High et al. (2012).

parameters from the shear profile fits, cf. Paper II; upper panel of Fig. 1), and 1.46 \pm 0.57 , if the B13 mass-concentration relation is applied (" $c_{\mathrm{B} 13}$ "; lower panel). The different slopes in the $c_{\mathrm{fit}}$ and $c_{\mathrm{B} 13}$ cases are mainly due to the two clusters, CL 1641+4001 and CL $1701+6414$, in which the weak lensing analysis revealed shallow tangential shear profiles due to extended surface mass plateaus (cf. Figs. 3 and 5 of Paper II). This will be the starting point for further analysis and interpretation in Sect. 4.1.
Although the $c_{\mathrm{B} 13}$ slope is consistent with the expected 1:1 relation, such a $M_{500}^{\mathrm{wl}}-M_{500}^{\text {hyd }}$ relation would translate to extreme biases between X-ray and WL masses if extrapolated to higher and lower masses. Especially for masses of a few $10^{15} M_{\odot}$, ample observations disagree with the extrapolated $M^{\text {wl }}>2 M^{\text {hyd }}$. We do not claim our data to have such predicting power outside its mass range. Rather, we focus on what can be learnt about the X-ray/WL mass bias in our $0.4 \sim z \sim 0.5$ mass 
range, which we, for the first time, study in the mass range down to $\sim 1 \times 10^{14} M_{\odot}$.

We are using three methods to test for the biases between $\mathrm{X}$-ray and WL masses. First, we compute the logarithmic bias $b=\langle\log \xi-\log \eta\rangle$, which we define as the average logarithmic difference between two general quantities $\xi$ and $\eta$. Its interpretation is that $10^{b} \eta$ is the average value corresponding to $\xi$. The uncertainty in $b$ is given by the standard error of $(\log \xi-\log \eta)$. Hence, our measurement of $b=-0.02 \pm 0.04$ for $c_{\mathrm{B} 13}$ corresponds to a vanishing fractional bias of $\left\langle M^{\text {hyd }}\right\rangle \approx$ $(0.97 \pm 0.09)\left\langle M^{\mathrm{wl}}\right\rangle$.

Given the small sample size, large uncertainties, and the tight scaling relations in Fig. 1 pointing to some correlation between the WL and X-ray masses, we base our further tests on a Monte Carlo (MC) analysis including the jackknife test. For $10^{5}$ realisations, we chose $\hat{\xi}_{i, k}=\xi_{i}+\delta \xi_{i, k}$ with random $\delta \xi_{i, k}$ drawn from zero-mean distributions assembled from two Gaussian halves with variances $\sigma_{\xi, i}^{-}$for the negative and $\sigma_{\xi, i}^{+}$for the positive half ${ }^{4}$. This provides a simple way of accommodating asymmetric uncertainties (cf. Paper II and Table 1). Then we take the logarithm and again symmetrise the errors. We repeat for $\hat{\eta}_{i, k}=\eta_{i}+\delta \eta_{i, k}$. On top, for each realisation $\left\{\hat{\xi}_{i, k}, \hat{\eta}_{i, k}\right\}$, we discard one cluster after another, yielding a total of $8 \times 10^{5}$ samples.

Based on those MC/jackknife realisations, we compute our second bias estimator $b_{\mathrm{MC}}=\langle\log \hat{\xi}-\log \hat{\eta}\rangle_{\mathrm{MC}}$. In order to achieve the best possible robustness against large uncertainties and small cluster numbers, we quote the ensemble median and dispersion. We find $b_{\mathrm{MC}}=0.00_{-0.13}^{+0.14}$ for $c_{\mathrm{B} 13}$, in good agreement with $b=-0.02 \pm 0.04$, i.e. a median WL/X-ray mass ratio of 1 .

Fitting $\log \left(M^{\mathrm{X}} / M^{\mathrm{wl}}\right)$ as a function of $M^{\mathrm{wl}}$ and averaging over the MC/jackknife samples, we obtain our third bias estimator, an intercept $A$ at the pivot mass of $\log \left(M_{\text {piv }} / \mathrm{M}_{\odot}\right)=14.5$. We find $A=-0.02_{-0.08}^{+0.07}$ for $c_{\mathrm{B} 13}$, again consistent with vanishing bias.

\subsection{Lensing masses and $X$-ray masses from proxies}

Figures 2 and A.2, as well as Table 2 present the three different X-ray/WL mass bias estimates for four X-ray mass observables: $M_{500}^{\text {hyd }}$ using $c_{\mathrm{B} 13}$ from Sect. 3.1 in Panel A of Fig. 2, $M_{500}^{\mathrm{Y}}$ in Panel B of Fig. 2, $M_{500}^{\mathrm{T}}$ in Panel A of Fig. A.2, $M_{500}^{\mathrm{G}}$ in Panel B of Fig. A.2. The last three are the proxy-based Vikhlinin et al. (2009a) X-ray mass estimators defined in Sect. 2.5. While Panel A uses $r_{500}^{\mathrm{wl}}$, the other three panel use the respective $r_{500}^{\mathrm{Y}, \mathrm{T}, \mathrm{G}}$.

Long-dashed lines and dark-grey boxes in Fig. 2 display $b$ and its error. Short-dashed lines and light-grey boxes denote $b_{\mathrm{MC}}$. The intercept $A$ is located at the intersection of the dot-dashed fit and dotted zero lines. We also show $b_{\mathrm{MC}}$ for the low-mass and high-mass clusters separately, splitting at $M^{\mathrm{wl}} E(z)=M_{\text {piv }}$; the respective $1 \sigma$ ranges are shown as outline boxes.

We observe remarkable agreement between the $\mathrm{X}$-ray/WL mass ratios and bias fits from all four X-ray observables (which are not fully independent). For each of them, all three bias estimates agree with each other, and all are consistent with no X-ray/WL mass bias. We find no evidence for X-ray masses being biased low by $\sim 40 \%$ in our cluster sample, as it has been suggested to explain the Planck CMB SZ cluster counts discrepancy. While $b_{\mathrm{MC}} \geq 0.2(\gtrsim 35 \%$ mass bias) lies within the possible range of the high- $M^{\mathrm{wl}}$ bin, in particular using the gas mass $M^{\mathrm{G}}$, the overall cluster sample

\footnotetext{
4 Unphysical cluster masses $<10^{13} M_{\odot} / E(z)$ are set to $10^{13} M_{\odot} / E(z)$.
}

does not support this hypothesis. We point out that $b_{\mathrm{MC}}$ was designed to be both robust against possible effects of large uncertainties and the small number of clusters in this first batch of $400 \mathrm{~d}$ WL clusters. The larger uncertainties in $b_{\mathrm{MC}}$ compared to $b$ are directly caused by the jackknife test and the account for possible fit instability in the MC method.

The slopes $B$ quantifying the $M^{\mathrm{wl}}$ dependence of the $\mathrm{X}$-ray/WL mass ratio are significantly negative in all of our measurements. This directly corresponds to the steep slope in the mass-mass scaling (Fig. 1). Predicting cluster masses for very massive clusters (or low-mass groups) from $B=-0.75$ for $M^{\mathrm{Y}}$ would yield $M^{\mathrm{X}}=M^{\mathrm{wl}} / 2$ at $10^{15} \mathrm{M}_{\odot} / E(z)$ (and $M^{\mathrm{X}}=$ $2.8 M^{\mathrm{wl}}$ at $\left.10^{14} M_{\odot} / E(z)\right)$. Such ratios are at odds with existing measurements. Therefore, we refrain from extrapolating cluster masses, but interpret the slopes $B$ as indicative of a possibly mass-dependent $\mathrm{X}$-ray/WL mass ratio. This evidence is more prudently presented as the $\sim 2 \sigma$ discrepancy between the low- $M^{\mathrm{wl}}$ and high- $M^{\mathrm{wl}}$ mass bins for all three V09a X-ray observables.

\section{A mass-dependent bias?}

In this section, we analyse two unexpected outcomes of our study in greater detail: the clear correlation between the $M^{\mathrm{X}} / M^{\mathrm{wl}}$ measurement of the individual clusters and their lensing masses, and the unusually small scatter in our scaling relations. Results for ancillary scaling relations that we present in Appendix A underpin the findings of Fig. 1 and Table 2. To begin with, we emphasise that the mass-dependent bias is not caused by the conflation of a large $z$ range; all but one of our clusters inhabit the range $0.39 \leq z \leq 0.53$ across which $E(z)$ varies by $<10 \%$, and we accounted for this variation. As Fig. A.2 shows, this also leaves us with little constraining power with regard to a $z$-dependent bias, at least until the $400 \mathrm{~d}$ WL survey becomes more complete.

\subsection{Role of $c_{200}$ and departures from NFW profile}

Figure 1 shows that the $M^{\mathrm{wl}}-M^{\mathrm{X}}$ scaling relation sensitively depends on the choice for the cluster concentration parameter $c_{200}$. This translates into more positive bias estimates for $c_{\text {fit }}$ as compared to $c_{\mathrm{B} 13}$ (Table 2). The difference is caused by the two flat-profile clusters for which NFW fits yield low masses but do not capture all the large-scale mass distribution, in particular if $c_{200}$ is determined directly from the data, rather than assuming a mass-concentration relation (cf. the discussion of the concentration parameter in Paper II and Foëx et al. 2012). This induces a bias towards low masses in the $r_{200} \rightarrow r_{500}$ conversion. If these two "irregular" clusters (dotted ring symbols in Fig. 1) are excluded, the "regular clusters only" $M^{\mathrm{wl}}-M^{\mathrm{X}}$ scaling relations (dash-dotted lines) differ for the $c_{\text {fit }}$, but not for the $c_{\mathrm{B} 13}$ case. Moreover, their mass ratios are consistent with the other high- $M^{\mathrm{wl}}$ clusters for $c_{\mathrm{B} 13}$. We thus confirm that assuming a mass-concentration relation and marginalising over $c_{200}$ is advantageous for scaling relations. We note that Comerford et al. (2010) observed a correlation between the scatters in the massconcentration and mass-temperature relations and advocated the inclusion of unrelaxed clusters in scaling relation studies.

Because NFW profile fits do not capture the complete projected mass morphologies of irregular clusters, the assumption of that profile for $M_{500}^{\mathrm{wl}}\left(r_{500}^{\mathrm{wl}}\right)(\mathrm{Eq} .3)$, and $M_{500}^{\mathrm{wl}}\left(r_{500}^{\mathrm{Y}}\right)$, etc. (Sect. 3.2) could introduce a further bias. Aperture-based lensing masses, e.g. the $\zeta_{\mathrm{c}}$ statistics (Clowe et al. 1998) employed by 
Hoekstra et al. (2012) provide an alternative. However, Okabe et al. (2013) demonstrated by the stacking of 50 clusters from the Local Cluster Substructure Survey $(0.15<z<0.3)$, that the average weak lensing profile does follow NFW to a high degree, at least at low redshift. Furthermore, the Planck Collaboration (2013) finds a trend of $M^{\mathrm{wl}} / M^{\text {hyd }}$ with the ratio of concentration parameters measured from weak lensing and X-rays.

\subsection{Correlation between mass estimates}

In Table 2 , we quote $\chi_{\text {red }}^{2}$ for the $M^{\mathrm{wl}}-M^{\mathrm{X}}$ scaling relations, using the same MC/jackknife samples as for the bias tests. For the ones involving hydrostatic masses, we measure $0.5<\chi_{\text {red }}^{2}<0.6$. We evaluated $M_{500}^{\text {hyd }}$ at $r_{500}^{\mathrm{wl}}$ in order to measure both estimates within the same physical radius, in an "apples with apples" comparison. But using the lensing-derived radius also introduces an unknown amount of correlation, a possible (partial) cause of the measured low $\chi_{\text {red }}^{2}$ values.

We test for the impact of the correlation by measuring both $M^{\mathrm{wl}}$ and $M^{\text {hyd }}$ within a fixed physical radius for all clusters, and choose $r_{\mathrm{fix}}=800 \mathrm{kpc}$ as a rough sample average of $r_{500}$. Surprisingly, we find an only slightly higher $\chi_{\text {red }}^{2}$, still $<1$ (see Table 2). As before, the bias estimators are consistent with zero. Fixed radii of $600 \mathrm{kpc}$ and $1000 \mathrm{kpc}$ give similar results (Table 2 and Fig. A.2). with a tentative trend of increasing $\chi_{\text {red }}^{2}$ with smaller radii. Interestingly, a low $\chi_{\text {red }}^{2}$ is also found for the $M^{\mathrm{wl}}$ $M^{\mathrm{T}}$ and $M^{\mathrm{T}}-M^{\text {hyd }}$ relations (Table A.2). The latter is expected, because $M^{\text {hyd }}$ are derived from the same $T_{\mathrm{X}}$ and depend sensitively on them. This all suggests that the small scatter is not driven by using $r_{500}^{\mathrm{wl}}$, but by some other intrinsic factor.

If the uncertainties in $M^{\mathrm{wl}}$ were overestimated significantly, this would obviously explain the low $\chi_{\text {red }}^{2}$. However, we do not even include systematic effects here. Moreover, the quoted $M^{\mathrm{wl}}$ uncertainties directly come from the NFW modelling of Paper II and reflect the $\Delta \chi^{2}$ from their Eq. (3), given the shear catalogue. The errors are dominated by the intrinsic source ellipticity $\sigma_{\varepsilon}$, for which we, after shear calibration, find values of $\sim 0.3$, consistent with other ground-based WL experiments. Therefore, despite the allure of our WL errors being overestimated, we do not find evidence for this hypothesis in our shear catalogues. Furthermore, the quoted $M^{\mathrm{wl}}$ uncertainties are consistent with the aperture mass detection significances we reported in Paper II.

\subsection{Dilution by cluster members and foregrounds}

Comparing the complete set of mass-mass scaling relations our data offer (Table A.2), we trace the mass-dependence of the bias seen in Fig. 2 back to the different ranges spanned by the estimates for $r_{500}$. While the ratio of minimum to maximum is $\approx 0.75$ for $r_{500}^{\mathrm{Y}}, r_{500}^{\mathrm{T}}$, and $r_{500}^{\text {hyd }}$, the same ratio is 0.57 for $r_{500}^{\mathrm{wl}}$, using the B13 $M-c$ relation. In the following, we discuss the potential influence of several sources of uncertainty in the WL masses, showing that the dispersion between lowest and highest $M_{500}^{\mathrm{wl}}$ is likely an inherent feature rather than a modelling artefact.

In Paper II, we discussed the great effort we took in constructing the best-possible homogeneous analysis from the quite heterogeneous MMT imaging data. Unfortunately, we happen to find higher $M^{\mathrm{wl}}$ for all clusters with imaging in three bands than for the clusters with imaging in one band. We emphasise there are no trends with limiting magnitude, seeing, or density $n_{\mathrm{KSB}}$ of galaxies with measurable shape (cf. Tables 1 and 2 in Paper II).
In the cases where three-band imaging is available, our WL model includes a correction for the diluting effect residual cluster member galaxies impose on the shear catalogue. For the other clusters, no such dilution correction could be applied. A rough estimate of the fraction of unlensed galaxies remaining after background selection suggests that the contamination in single-band catalogues is $\sim 30-50 \%$ higher than with the more sophisticated galaxy-colour based method. Therefore, we recalculate the scaling relations, switching off the dilution correction (long dashed line and thin ring symbols in Fig. 1). This lowers the $r_{500}$ values by $\sim 6 \%$ and the masses by $10-15 \%$. For both the $M_{500}^{\mathrm{wl}}-M_{500}^{\text {hyd }}$ and $M_{500}^{\mathrm{wl}}-M_{500}^{\mathrm{Y}}$ relations, we only observe a slightly smaller difference between $b_{\mathrm{MC}}$ for the high- and low$M^{\mathrm{wl}}$ bins (Tables 2 and A.2), not significant given the uncertainty margins.

The dilution of the shear signal by an increased number of galaxies not bearing a shear signal can also be expressed as a overestimation of the mean lensing depth $\langle\beta\rangle$. We model a possible lensing depth bias by simultaneously adding the uncertainty $\sigma(\langle\beta\rangle)$ for the three-band clusters and subtracting it for the single-band ones, maximising the leveraging effect on the masses. Similar to the previous experiment ${ }^{5}$ we still observe a mass-dependent bias, with little change to the default model.

We further tested alternative choices of cluster centre and fitting range, but do not observe significant changes to the mass dependent-bias or to $\chi_{\text {red }}^{2}$ (see Appendix A.3). Although $\langle\beta\rangle$ is calculated for all clusters from the same catalogues, related systematics would affect the mass normalisation, but not the relative stochastic uncertainties, which determine $\chi_{\text {red }}^{2}$. As we consider a drastic overestimation of the purely statistic uncertainties in the WL modelling being unlikely (Sect. 4.2), the cause of the low $\chi_{\text {red }}^{2}$ values remains elusive. If a WL analysis effect is responsible for one or both anomalies, it has to be of a more subtle nature than the choices investigated here.

\subsection{A statistical fluke?}

We summarise that the $M_{500}^{\mathrm{wl}}-M_{500}^{\text {hyd }}$ scaling relations we observe show an unusual lack of scatter and that we find a $\sim 2 \sigma$ difference between the X-ray/WL mass ratios of our high- and low- $M^{\mathrm{wl}}$ clusters. The latter effect can be traced back to the considerable span in cluster lensing signal, which is only partly due to different background selection procedures and the dilution correction that was only applied for clusters imaged in three bands.

The question then arises if the mass-dependent bias is caused by an unlucky selection of the 8 MMT clusters from V09's wider sample of 36 . The 8 clusters were chosen to be observed first merely because of convenient telescope scheduling, and appear typical of the larger sample in terms of redshift and X-ray observables. The MMT clusters trace well the mass range and dispersion spanned by all 36 clusters in their $M_{500}^{\mathrm{Y}}-M_{500}^{\mathrm{T}}$ relation. We observe the expected vanishing slopes for $\log \left(M^{\mathrm{T}} / M^{\mathrm{Y}}\right)$ as a function of $M^{\mathrm{Y}}$, both for the 8 MMT clusters and for the complete sample of 36 (Table A.2).

In Table 2, we observe significant scatter $\left(\chi_{\text {red }}^{2}=2.11\right)$ in the $M_{500}^{\mathrm{wl}}-M_{500}^{\mathrm{G}}$ relation, while Okabe et al. (2010b) and M 13 reported particularly low scatter in $M^{\mathrm{G}}$, comparing to WL masses. This large intrinsic scatter seems to be a feature of the overall 400d sample: plotting $M^{\mathrm{G}}$ versus the two other V09 X-ray

\footnotetext{
5 Because an unnoticed higher dilution in the catalogue does not imply a bias in the estimation of $\langle\beta\rangle$ from a proxy catalogue, the two effects are not likely to add up.
} 


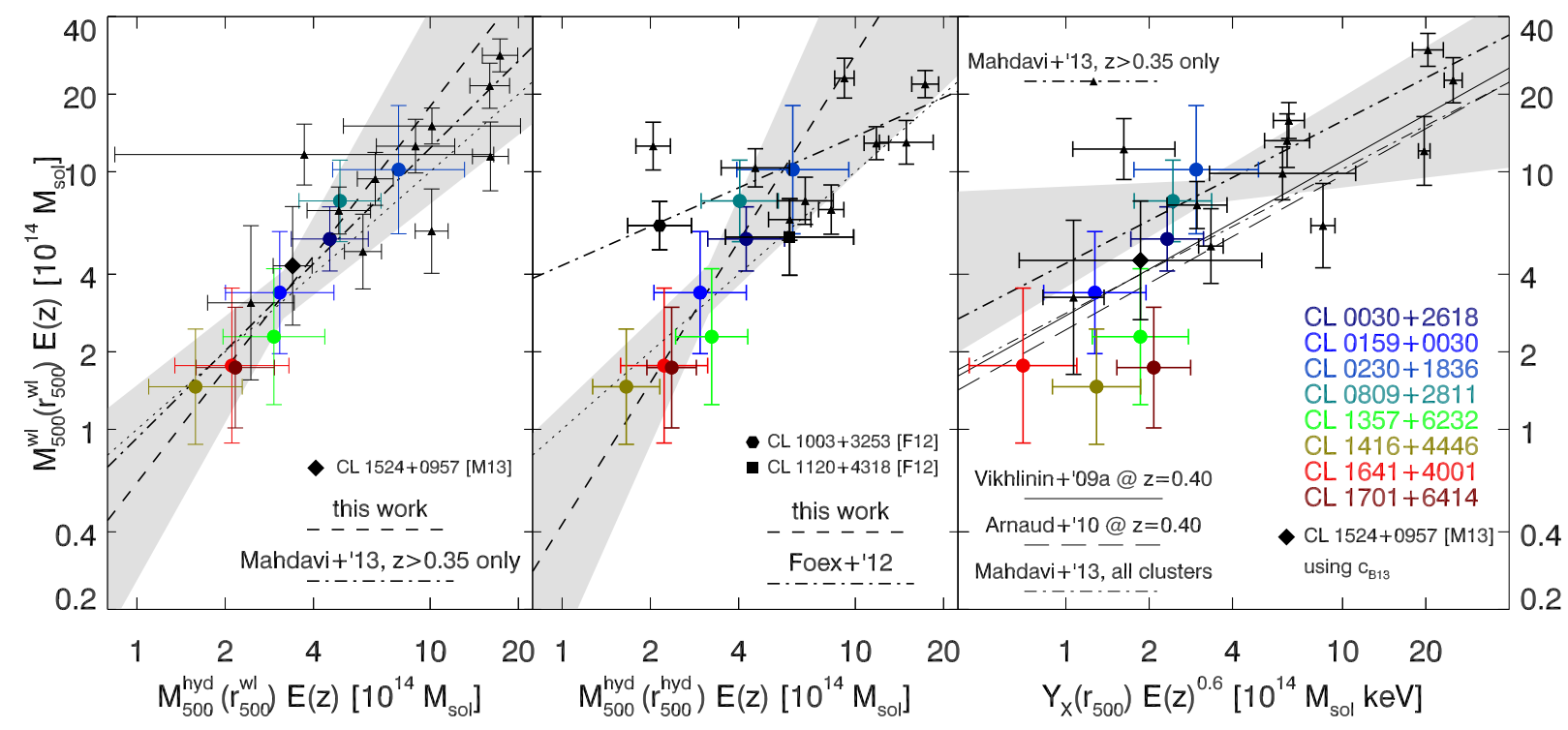

Fig. 3. Comparisons with literature data. Left panel: black symbols show $z>0.35$ clusters from Mahdavi et al. (2013), whose best-fit using Eq. (5) is shown by the dot-dashed line. The cluster CL $1524+0957$ is indicated by a diamond symbol. Coloured symbols and the dashed line show the "default" $M_{500}^{\mathrm{wl}}-M_{500}^{\mathrm{hyd}}$ relation for $c_{\mathrm{B} 13}$ as in the lower panel of Fig. 1. Middle panel: the same, but comparing to Foëx et al. (2012) (black symbols and dot-dashed line for best fit). X-ray masses are measured within $r_{500}^{\text {hyd }}$. CL 1003+3253 and CL 1120+4318 are emphasised by special symbols. Right panel: scaling of lensing masses $M_{500}^{\mathrm{wl}}$ with the $Y_{\mathrm{X}}$ proxy. Black symbols show the $z>0.35$ clusters from M13, to which the thick, dash-dotted line is the best fit. Shaded regions indicate the uncertainties to this fit. The thin, dash-dotted line gives the best fit M13 quote for their complete sample, while the thin solid and long-dashed lines mark the $M_{500}-Y_{\mathrm{X}}$ relations by V09a and Arnaud et al. (2010), respectively, for $z=0.40$.

masses of all 36 clusters, we also find $\chi_{\text {red }}^{2}>2$ (Table A.2), as well as significant non-zero logarithmic biases. While tracing the cause of this observation is beyond the scope of this article, it deserves further study. Because two of the clusters with highest $\left|M_{500}^{\mathrm{Y}, \mathrm{T}}-M_{500}^{\mathrm{G}}\right|$ are covered by our MMT subsample, we observe a more mass-dependent $M^{\mathrm{G}} / M^{\mathrm{Y}, \mathrm{T}}$ ratio than for all 36. Overall, however, the MMT subsample is not a very biased selection.

\subsection{Physical causes}

An alternative and likely explanation for the mass-dependent bias we observe could be a high rate of unrelaxed clusters, especially for our least massive objects. If the departure from hydrostatic equilibrium were stronger among the low-mass clusters than for the massive ones, this would manifest in mass ratios similar to our results. Simulations show the offset from hydrostatic equilibrium to be mass-dependent (Rasia et al. 2012), despite currently being focused on the high-mass regime. Variability in the non-thermal pressure support with mass (Laganá et al. 2013) may be exacerbated by small number statistics. At high $z$, the expected fraction of merging clusters, especially of major mergers, increases. Unrelaxed cluster states are known to affect X-ray observables and, via the NFW fitting, also lensing mass estimates. Indeed, the two most deviant systems in Fig. 2 are CL $1416+4446$ and the flat-profile "shear plateau" cluster CL 1641+4001. Although the first shows an inconspicuous shear profile, we suspect it to be part of a possibly interacting supercluster, based on the presence of two nearby structures at the same redshift, detected in X-ray as well as in our lensing maps (Paper II). Both these clusters are classified as non-mergers in the recent Nurgaliev et al. (2013) study, introducing a new substructure estimator based on X-ray morphology. However, WL and X-ray methods are sensitive to substructure on different radial and mass scales, such that this explanation cannot be ruled out. We summarise that the greater dynamical range in WL than in X-ray masses might be linked to different sensitivities of the respective methods to substructure and mergers in the low-mass, high- $z$ cluster population we are probing, but which is currently still underexplored.

\section{Comparison with previous works}

\subsection{The $M_{500}^{\mathrm{wl}}-M_{500}^{\text {hyd }}$ relation}

Comparison with Mahdavi et al. (2013) results: recently, M13 published scaling relations observed between the weak lensing and X-ray masses for a sample of 50 massive clusters, partly based on the brightest clusters from the Einstein Observatory Extended Medium Sensitivity Survey (Gioia et al. 1990). Weak lensing masses for the M13 sample have been obtained from CFHT/Megacam imaging (Hoekstra et al. 2012), while the X-ray analysis combines XMM-Newton and Chandra data. While the median redshift is $z=0.23$, the distribution extends to $z=0.55$, including 12 clusters at $z>0.35$. Owing to their selection, these 12 clusters lie above the $400 \mathrm{~d}$ flux and luminosity cuts, making them directly comparable to our sample.

The left panel in Fig. 3 superimposes the $M_{500}^{\mathrm{wl}}$ and $M_{500}^{\text {hyd }}$ of the M13 high- $z$ clusters on our results. The two samples overlap at the massive $\left(\gtrsim 5 \times 10^{14} M_{\odot}\right)$ end, but the $400 \mathrm{~d}$ objects probe down to $1 \times 10^{14} M_{\odot}$ for the first time at this $z$ and for these scaling relations. The slopes of the scaling relations are consistent: using Eq. (5), we measure $B_{\mathrm{M}-\mathrm{M}}=1.13 \pm 0.20$ for the $12 \mathrm{M} 13$ objects. A joint fit with the $400 \mathrm{~d}$ clusters $\left(B_{\mathrm{M}-\mathrm{M}}=1.46 \pm 0.57\right)$ yields $B_{\mathrm{M}-\mathrm{M}}=1.15 \pm 0.14$ and a low $\chi_{\text {red }}^{2}=0.54$, driven by our data. We note that the logarithmic bias of $b=0.10 \pm 0.05$ for the M13 high- $z$ clusters corresponds to a $(20 \pm 10) \%$ mass bias, consistent with both the upper range of the $400 \mathrm{~d}$ results and 
expectation from the literature (e.g. Laganá et al. 2010; Rasia et al. 2012).

Calculating the Hogg et al. (2010, H10) likelihood which Mahdavi et al. (2013) use, we find $B_{\mathrm{H} 10}=1.18_{-0.20}^{+0.22}$ and intrinsic scatter $\sigma_{\text {int }}$ consistent with zero, confirming our above results. If we, however, repeating our fits from Fig. 1 with the H10 likelihood, we obtain discrepant results which highlight the differences between the various regression algorithms (see Sect. 2.6) ${ }^{6}$.

CL $1524+0957$ at $z=0.52$ is the only cluster the $400 \mathrm{~d}$ and M13 samples share. Denoted by a black diamond in Fig. 3, its masses from the M13 lensing and hydrostatic analyses blend in with the MMT 400d clusters. If it were included in the $M_{500}^{\mathrm{wl}}-$ $M_{500}^{\text {hyd }}$ relation, it would not significantly alter the best fit, but we caution that different analysis methods have been employed, e.g. M13 reporting aperture lensing masses based on the $\zeta_{\mathrm{c}}$ statistics.

Comparison with Jee et al. (2011) results: Jee et al. (2011, J11) studied 14 very massive and distant clusters $(0.83<z<$ 1.46) and found their WL and hydrostatic masses $M_{200}^{\mathrm{wl}}$ and $M_{200}^{\text {hyd }}$ to agree well, similar to our results. However, they caution that their $M_{200}^{\text {hyd }}$ were obtained by extrapolating a singular isothermal sphere profile. Because we doubt that the Chandra-based Vikhlinin et al. (2006) model reliably describes the ICM out to such large radii, we refrain from deriving $M_{200}^{\text {hyd }}$. Nevertheless, we notice that our the J11 samples not only shows similar $M_{200}^{\mathrm{wl}}$ than our most massive clusters, but also contains the only two 400d clusters exceeding the redshift of CL $0230+1836$, CL 0152-1357 at $z=0.83$ and CL $1226+3332$ at $z=0.89$. Their planned re-analysis will improve the leverage of our samples at the high- $z$ end.

Comparison with Foëx et al. (2012) results: in the middle panel of Fig. 3, we compare our results to 11 clusters from the EXCPRES XMM-Newton sample, analysed by Foëx et al. (2012, F12) and located at a similar redshift range $(0.41 \leq z \leq 0.61)$ as the bulk of our sample. Selected to be representative of the cluster population at $z \approx 0.5$, these objects have been studied with XMM-Newton in X-rays and CFHT/Megacam in the optical. Foëx et al. (2012) explicitly quote hydrostatic and lensing masses within their respective radii; thus we also show the $M_{500}^{\mathrm{wl}}\left(r_{500}^{\mathrm{wl}}\right)-M_{500}^{\mathrm{hyd}}\left(r_{500}^{\mathrm{hyd}}\right)$. Again, the more massive of our clusters resemble the F12 sources, with the 400d MMT sample extending towards lower masses. Indeed, F12 study two clusters which are part of our sample: these, CL $1002+3253$ at $z=0.42$ and CL $1120+4318$ at $z=0.60$ mark their lowest lensing mass objects. At similar $M_{500}^{\mathrm{wl}}$ on either sides of the best-fit $400 \mathrm{~d}$ scaling relation, their inclusion with the quoted masses would have no immediate effect on its slope, but slightly increase its scatter.

Bearing in mind that Fig. 3 (middle panel) compares quantities measured at different radii, we notice that the significantly flat best fit regression line to the F12 cluster masses, showing a larger dispersion in hydrostatic than in WL masses, as opposed to the 400d MMT clusters. The comparisons in Fig. 3 underscore that while being broadly compatible with each other, different studies are shaped by the fine details of their sample

\footnotetext{
6 In fact, regression lines not only depend on the likelihood or definition of the best fit, but also on the algorithm used to find its extremum, and, if applicable, how uncertainties are transferred from the linear to the logarithmic domain. Thus, our H10 slopes agree with the ones the web-tool provided by M13 yield, but produce different uncertainties.
}

selection and analysis methods. We will conduct a more detailed comparison between our results and the ones of Foëx et al. (2012) and Mahdavi et al. (2013) once we re-analysed the CFHT/Megacam of the three overlapping clusters, having already shown the MMT and CFHT Megacams to produce consistent lensing catalogues (Paper II).

\subsection{The $M_{500}^{\mathrm{wl}}-M_{500}^{\mathrm{Y}}$ relation}

The right panel of Fig. 3 investigates the scaling behaviour of $M_{500}^{\mathrm{wl}}$ with $Y_{\mathrm{X}}$ by comparing the $400 \mathrm{~d}$ MMT clusters to the $z>0.35$ clusters from $\mathrm{M} 13^{7}$. The difference between the two samples is more pronounced than in the left panel, with only the low-mass end of the M13 sample, including CL 1524+0957, overlapping with our clusters. None of the 400d MMT clusters deviates significantly from the $M_{500}-Y_{\mathrm{X}}$ relation applied by V09a in the derivation of the $M_{500}^{\mathrm{Y}}$ masses we used. The V09a $M_{500}-Y_{\mathrm{X}}$ relation based on Chandra data for low- $z$ clusters (Vikhlinin et al. 2006) is in close agreement to the M13 result for their complete sample, as well as the widely used Arnaud et al. (2010) $M_{500}-Y_{\mathrm{X}}$ relation. For the latter as well as V09a we show the version with a slope fixed to the self-similar expectation of $B=3 / 5$. The best fit to the M13 $z>0.35$ essentially yields the same slope as the complete sample $(B=0.55 \pm 0.09$ compared to $B_{\mathrm{H} 10}=0.56 \pm 0.07$, calculated with the $\mathrm{H} 10$ method). The higher normalisation for the high- $z$ subsample can be likely explained as Malmquist bias due to the effective higher mass limit in the M13 sample selection. The incompatibility of the least massive MMT clusters with this fit highlights that we sample lower mass clusters, which, at the same redshift, are likely to have different physical properties.

The $Y_{\mathrm{X}}$ proxy is the $\mathrm{X}$-ray equivalent to the integrated pressure signal $Y_{\mathrm{SZ}}$ seen by $\mathrm{SZ}$ observatories. Observations confirm a close $Y_{\mathrm{X}}-Y_{\mathrm{SZ}}$ correlation, with measured departures from the 1:1 slope considered inconclusive (Andersson et al. 2011; Rozo et al. 2012). Performing a cursory comparison with SZ observations, we included in Fig. 2A data for three $z>0.35$ clusters from High et al. (2012) (dashed uncertainty bars), taken from their Fig. 6. The abscissa values for the High et al. (2012) clusters (SPT-CL J2022-6323, SPT-CL J2030-5638, and SPT-CL J2135-5726) show masses based on $Y_{\mathrm{SZ}}$, derived from South Pole Telescope SZ observations (Reichardt et al. 2013). The $M^{\mathrm{wl}}$ are derived from observations with the same Megacam instrument we used for the 400d clusters, but after its transfer to the Magellan Clay telescope at Las Campanas Observatory, Chile. In good agreement with zero bias, the High et al. (2012) clusters are consistent with some of the lower mass 400d clusters. This result suggests that the $Y_{\mathrm{X}}-Y_{\mathrm{SZ}}$ equivalence might hold once larger samples at high $z$ and low masses will become available.

\section{Summary and conclusions}

In this article, we analysed the scaling relation between WL and $\mathrm{X}$-ray masses for 8 galaxy clusters drawn from the 400d sample of X-ray-luminous $0.35 \leq z \leq 0.89$ clusters. WL masses were measured from the Israel et al. (2012) MMT/Megacam data, and X-ray masses were based on the V09a Chandra analysis. We summarise our main results as follows:

\footnotetext{
7 Owing to the availability of data, we need to use different definitions of $r_{500}$ for the two data sets.
} 
1. We probe the WL-X-ray mass scaling relation, in an unexplored region of the parameter space for the first time: the $z \sim 0.4-0.5$ redshift range, down to $1 \times 10^{14} M_{\odot}$.

2. Using several X-ray mass estimates, we find the WL and $\mathrm{X}$-ray masses to be consistent with each other. Most of our clusters are compatible with the $M^{\mathrm{X}}=M^{\mathrm{wl}}$ line.

3. Assuming the $M^{\mathrm{wl}}$ not to be significantly biased, we do not find evidence for a systematic underestimation of the X-ray masses by $\sim 40 \%$, as suggested as a possible solution to the discrepancy between the Planck CMB constraints on $\Omega_{\mathrm{m}}$ and $\sigma_{8}$ (the normalisation of the matter power spectrum) and the Planck SZ cluster counts (Planck Collaboration XX 2014). While our results favour a small WL-X-ray mass bias, they are consistent with both vanishing bias and the $\sim 20 \%$ favoured by studies of non-thermal pressure support.

4. For the mass-mass scaling relations involving $M^{\text {wl }}$, we observe a surprisingly low scatter $0.5<\chi_{\text {red }}^{2}<0.6$, although we use only stochastic uncertainties and allow for correlated errors via a Monte Carlo method. Because the errors in $M^{\mathrm{wl}}$ are largely determined by the intrinsic WL shape noise $\sigma_{\varepsilon}$, we however deem a drastic overestimation unlikely (Sect. 4.2). For the scaling relations involving $M_{\mathrm{G}}$, however, we observe a large scatter, contrary to Okabe et al. (2010b) and M13.

5. Looking in detail, there are intriguing indications for a massdependence of the WL-X-ray mass ratios of our relatively low-mass $z \sim 0.4-0.5$ clusters. We observe a mass bias in the low $-M^{\mathrm{wl}}$ mass bin at the $\sim 2 \sigma$ level when splitting the sample at $\log \left(M_{\text {piv }} / M_{\odot}\right)=14.5$ This holds for the masses V09a report based on the $Y_{\mathrm{X}}, T_{\mathrm{X}}$, and $M_{\mathrm{G}}$ proxies.

The discrepant temperatures Chandra and XMM-Newton measure in clusters (Nevalainen et al. 2010; Schellenberger et al. 2012) could provide a possible avenue to reconcile Planck cluster properties with the Planck cosmology.

We thoroughly investigate possible causes for the massdependent bias and tight scaling relations. First (Sect. 4.1), we confirm that by using a mass-concentration relation instead of directly fitting $c_{200}$ from WL, we already significantly reduced the bias due to conversion from $r_{200}$ to $r_{500}$. We emphasise that, on average, the NFW shear profile represents a suitable fit for the cluster population (cf. Okabe et al. 2013). Measuring $M^{\text {hyd }}$ within $r_{500}^{\mathrm{wl}}$ induces correlation between the data points in Fig. 1. Removing this correlation by plotting both masses within a fixed physical radius, we still find small scatter (Sect. 4.2).

We notice that the mass range occupied by the $M^{\text {wl }}$ exceeds the X-ray mass ranges. Partially, this higher WL mass range can be explained by the correction for dilution by member galaxies, which could be applied only where colour information was available (Paper II). Coincidentally, this is the case for the more massive half of the MMT sample in terms of $M^{\mathrm{wl}}$, thus boosting the range of measured WL masses (Sect. 4.3). This result underscores the importance of correcting for the unavoidable inhomogeneities in WL data due to the demanding nature of WL observations (cf. Applegate et al. 2014). We find no further indications for biases via the WL analysis. Furthermore the tight scaling precludes strong redshift effects, and we find that our small MMT subsample is largely representative of the complete sample of 36 clusters, judging from the $M^{\mathrm{Y}}-M^{\mathrm{T}}$ relation (Sect. 4.4). For the $M^{\mathrm{Y}}-M^{\mathrm{G}}$ and $M^{\mathrm{T}}-M^{\mathrm{G}}$ relations, significant scatter $\left(\chi_{\text {red }}^{2}>2\right)$ is present in the larger sample. The former relation also shows indications for a significant bias of $M^{\mathrm{Y}} \approx 1.15 M^{\mathrm{G}}$.

Weak lensing and hydrostatic masses for the 400d MMT clusters are in good agreement with the $z>0.35$ part of the Mahdavi et al. (2013) sample and the $M_{500}^{\mathrm{wl}}-M_{500}^{\text {hyd }}$ relation derived from it (Sect. 5.1). The M13 and Foëx et al. (2012) samples include three 400d clusters with CFHT WL masses. These clusters neither point to significantly higher scatter nor to a less mass-dependent bias (Fig. 3). We are planning a re-analysis of the CFHT data, having demonstrated in Paper II that lensing catalogues from MMT and CFHT are nicely compatible. Such reanalysis is going to be helpful to identify more subtle WL analysis effects potentially responsible for the steep slopes and tight correlation of WL and X-ray masses.

An alternative explanation are intrinsic differences in the low-mass cluster population. That the 400d MMT sample probes to slightly lower masses $\left(1 \times 10^{14} M_{\odot}\right)$ than M13 or F12 becomes especially obvious from the $M^{\mathrm{wl}}-Y^{\mathrm{X}}$ relation (Fig. 3, Sect. 5.2). Because the $400 \mathrm{~d}$ sample is more representative of the $z \sim 0.4-0.5$ cluster population, it is likely to contain more significant mergers relative to the cluster mass, skewing mass estimates (Sect. 4.5). Hence, the 400d survey might be the first to see the onset of a mass regime in which cluster physics and substructure lead the WL-X-ray scaling to deviate from what is known at higher masses. Remarkably, Giles et al. (2014) are finding a different steep slopes in their low-mass WL-X-ray scaling analysis. Detailed investigations of how their environment shapes clusters like CL $1416+4446$ might be necessary to improve our understanding of the cluster population to be seen by future cosmology surveys. Analysis systematics might also behave differently at lower masses. A turn for WL cluster science towards lower mass objects, e.g. through the completion of the 400d WL sample, will help addressing the question of evolution in lensing mass scaling relations.

Acknowledgements. The authors express their thanks to M. Arnaud and EXCPRES collaboration (private communication) for providing the hydrostatic masses of the Foëx et al. (2012) clusters. We further thank A. Mahdavi for providing the masses of the Mahdavi et al. (2013) clusters via their helpful online interface. H.I. likes to thank M. Klein, J. Stott, and Y.-Y. Zhang, and the audiences of his presentations for useful comments. The authors thank the anonymous referee for their constructive suggestions. H.I. acknowledges support for this work has come from the Deutsche Forschungsgemeinschaft (DFG) through Transregional Collaborative Research Centre TR 33 as well as through the Schwerpunkt Program 1177 and through European Research Council grant MIRG-CT-208994. T.H.R. acknowledges support by the DFG through Heisenberg grant RE $1462 / 5$ and grant RE $1462 / 6$. T.E. is supported by the DFG through project ER 327/3-1 and by the Transregional Collaborative Research Centre TR 33 "The Dark Universe". R.M. is supported by a Royal Society University Research Fellowship. We acknowledge the grant of MMT observation time (program 2007B-0046) through NOAO public access. MMT time was also provided through support from the F. H. Levinson Fund of the Silicon Valley Community Foundation.

Note added in proof. After this paper was accepted, another paper (von der Linden et al. 2014b) appeared as submitted, pointing at the combined effects of hydrostatic mass bias and calibration systematics. The cross calibration effect on cosmological parameter constraints is currently being tested directly by Schellenberger et al. (in prep.). Rozo et al. (2014a,b) review and cross-calibrate the various scaling relations involved. Alternatively, Burenin (2013) suggested additional massive neutrino species.

\section{References}

Akritas, M. G., \& Bershady, M. A. 1996, ApJ, 470, 706

Allen, S. W., Evrard, A. E., \& Mantz, A. B. 2011, ARA\&A, 49, 409 Amendola, L., Appleby, S., Bacon, D., et al. 2013, Liv. Rev. Rel., 16, 6 Andersson, K., Benson, B. A., Ade, P. A. R., et al. 2011, ApJ, 738, 48 Andreon, S., \& Hurn, M. A. 2012 [arXiv: 1210.6232]

Applegate, D. E., von der Linden, A., Kelly, P. L., et al. 2014, MNRAS, 439, 48 Arnaud, M., Pratt, G. W., Piffaretti, R., et al. 2010, A\&A, 517, A92 
Bahé, Y. M., McCarthy, I. G., \& King, L. J. 2012, MNRAS, 421, 1073

Bartelmann, M. 1996, A\&A, 313, 697

Becker, M. R., \& Kravtsov, A. V. 2011, ApJ, 740, 25

Bharadwaj, V., Reiprich, T. H., Schellenberger, G., et al. 2014, A\&A, accepted [arXiv: 1402.0868]

Bhattacharya, S., Habib, S., Heitmann, K., \& Vikhlinin, A. 2013, ApJ, 766, 32 (B13)

Bullock, J. S., Kolatt, T. S., Sigad, Y., et al. 2001, MNRAS, 321, 559 (B01)

Burenin, R. A. 2013, Astron. Lett., 39, 357

Burenin, R. A., Vikhlinin, A., Hornstrup, A., et al. 2007, ApJS, 172, 561

Cavaliere, A., \& Fusco-Femiano, R. 1978, A\&A, 70, 677

Clowe, D., Luppino, G. A., Kaiser, N., Henry, J. P., \& Gioia, I. M. 1998, ApJ, 497, L61

Comerford, J. M., Moustakas, L. A., \& Natarajan, P. 2010, ApJ, 715, 162

Corless, V. L., \& King, L. J. 2009, MNRAS, 396, 315

Croston, J. H., Pratt, G. W., Böhringer, H., et al. 2008, A\&A, 487, 431

Dietrich, J. P., Erben, T., Lamer, G., et al. 2007, A\&A, 470, 821

Dietrich, J. P., Böhnert, A., Lombardi, M., Hilbert, S., \& Hartlap, J. 2012, MNRAS, 419, 3547

Eckmiller, H. J., Hudson, D. S., \& Reiprich, T. H. 2011, A\&A, 535, A105

Erben, T., van Waerbeke, L., Bertin, E., Mellier, Y., \& Schneider, P. 2001, A\&A, 366,717

Erben, T., Schirmer, M., Dietrich, J. P., et al. 2005, Astron. Nachr., 326, 432

Ettori, S. 2013, Astron. Nachr., 334, 354

Ettori, S., Donnarumma, A., Pointecouteau, E., et al. 2013, Space Sci. Rev., 177, 119

Feigelson, E., \& Babu, G. 2012, Modern Statistical Methods for Astronomy: With R Applications (Cambridge University Press)

Foëx, G., Soucail, G., Pointecouteau, E., et al. 2012, A\&A, 546, A106 (F12)

George, M. R., Leauthaud, A., Bundy, K., et al. 2012, ApJ, 757, 2

Giles, P. A., Maughan, B. J., Hamana, T., et al. 2014 [arXiv: 1402 . 4484]

Giodini, S., Lovisari, L., Pointecouteau, E., et al. 2013, Space Sci. Rev., 177, 247

Gioia, I. M., Maccacaro, T., Schild, R. E., et al. 1990, ApJS, 72, 567

Hartlap, J., Schrabback, T., Simon, P., \& Schneider, P. 2009, A\&A, 504, 689

Heymans, C., van Waerbeke, L., Bacon, D., et al. 2006, MNRAS, 368, 1323

High, F. W., Hoekstra, H., Leethochawalit, N., et al. 2012, ApJ, 758, 68

Hildebrandt, H., Erben, T., Dietrich, J. P., et al. 2006, A\&A, 452, 1121

Hoekstra, H. 2007, MNRAS, 379, 317

Hoekstra, H., Donahue, M., Conselice, C. J., McNamara, B. R., \& Voit, G. M. 2011a, ApJ, 726, 48

Hoekstra, H., Hartlap, J., Hilbert, S., \& van Uitert, E. 2011b, MNRAS, 412, 2095 Hoekstra, H., Mahdavi, A., Babul, A., \& Bildfell, C. 2012, MNRAS, 427, 1298 Hoekstra, H., Bartelmann, M., Dahle, H., et al. 2013, Space Sci. Rev., 177, 75

Hogg, D. W., Bovy, J., \& Lang, D. 2010, unpublished

[arXiv: 1008.4686] (H10)

Ilbert, O., Arnouts, S., McCracken, H. J., et al. 2006, A\&A, 457, 841

Israel, H., Erben, T., Reiprich, T. H., et al. 2010, A\&A, 520, A58 (Paper I)

Israel, H., Erben, T., Reiprich, T. H., et al. 2012, A\&A, 546, A79 (Paper II)

Jee, M. J., Dawson, K. S., Hoekstra, H., et al. 2011, ApJ, 737, 59 (J11)

Kaiser, N. 1986, MNRAS, 222, 323

Kaiser, N., Squires, G., \& Broadhurst, T. 1995, ApJ, 449, 460

Kelly, B. C. 2007, ApJ, 665, 1489

Laganá, T. F., de Souza, R. S., \& Keller, G. R. 2010, A\&A, 510, A76

Laganá, T. F., Martinet, N., Durret, F., et al. 2013, A\&A, 555, A66

Laureijs, R., Amiaux, J., Arduini, S., et al. 2011

[arXiv: 1110.3193]

Limousin, M., Morandi, A., Sereno, M., et al. 2013, Space Sci. Rev., 177, 155

Mahdavi, A., Hoekstra, H., Babul, A., \& Henry, J. P. 2008, MNRAS, 384, 1567

Mahdavi, A., Hoekstra, H., Babul, A., et al. 2013, ApJ, 767, 116, (M13)

Markwardt, C. B. 2009, in Astronomical Data Analysis Software and Systems XVIII, eds. D. A. Bohlender, D. Durand, \& P. Dowler, ASP Conf. Ser., 411,251

Marrone, D. P., Smith, G. P., Richard, J., et al. 2009, ApJ, 701, L114

Marrone, D. P., Smith, G. P., Okabe, N., et al. 2012, ApJ, 754, 119

Massey, R., Hoekstra, H., Kitching, T., et al. 2013, MNRAS, 429, 661

Maughan, B. J. 2014, MNRAS, 437, 1171
Maughan, B. J., Giles, P. A., Randall, S. W., Jones, C., \& Forman, W. R. 2012, MNRAS, 421, 1583

Merloni, A., Predehl, P., Becker, W., et al. 2012

[arXiv: 1209.3114]

Miyatake, H., Nishizawa, A. J., Takada, M., et al. 2013, MNRAS, 429, 3627

Nandra, K., Barret, D., Barcons, X., et al. 2013 [arXiv: 1306.2307]

Navarro, J. F., Frenk, C. S., \& White, S. D. M. 1996, ApJ, 462, 563

Navarro, J. F., Frenk, C. S., \& White, S. D. M. 1997, ApJ, 490, 493

Nevalainen, J., David, L., \& Guainazzi, M. 2010, A\&A, 523, A22

Newman, A. B., Treu, T., Ellis, R. S., et al. 2013, ApJ, 765, 24

Nurgaliev, D., McDonald, M., Benson, B. A., Stubbs, C. W., \& Vikhlinin, A. 2013, ApJ, 779, 112

Oguri, M., \& Hamana, T. 2011, MNRAS, 414, 1851

Okabe, N., Takada, M., Umetsu, K., Futamase, T., \& Smith, G. P. 2010a, PASJ, 62,811

Okabe, N., Zhang, Y.-Y., Finoguenov, A., et al. 2010b, ApJ, 721, 875

Okabe, N., Smith, G. P., Umetsu, K., Takada, M., \& Futamase, T. 2013, ApJ, 769, L35

Pacaud, F., Pierre, M., Adami, C., et al. 2007, MNRAS, 382, 1289

Pillepich, A., Porciani, C., \& Reiprich, T. H. 2012, MNRAS, 422, 44

Planck Collaboration 2013, A\&A, 550, A129

Planck Collaboration XX. 2014, A\&A, in press DOI: 10.1051/0004-6361/201321521

Pointecouteau, E., Reiprich, T. H., Adami, C., et al. 2013 [arXiv: 1306.2319]

Postman, M., Coe, D., Benítez, N., et al. 2012, ApJS, 199, 25

Predehl, P., Andritschke, R., Böhringer, H., et al. 2010, in SPIE Conf. Ser., 7732

Press, W. H., Teukolsky, S. A., Vetterling, W. T., \& Flannery, B. P. 1992, Numerical recipes in C. The art of scientific computing, 2nd ed. (Cambridge: University Press)

Rasia, E., Meneghetti, M., Martino, R., et al. 2012, New J. Phys., 14, 055018

Reese, E. D., Mroczkowski, T., Menanteau, F., et al. 2012, ApJ, 751, 12

Reichardt, C. L., Stalder, B., Bleem, L. E., et al. 2013, ApJ, 763, 127

Reichert, A., Böhringer, H., Fassbender, R., \& Mühlegger, M. 2011, A\&A, 535, A4

Reiprich, T. H., Basu, K., Ettori, S., et al. 2013, Space Sci. Rev., 195

Rozo, E., Vikhlinin, A., \& More, S. 2012, ApJ, 760, 67

Rozo, E., Bartlett, J. G., Evrard, A. E., \& Rykoff, E. S. 2014a, MNRAS, 438, 78 Rozo, E., Rykoff, E. S., Bartlett, J. G., \& Evrard, A. 2014b, MNRAS, 438, 49

Sarazin, C. L. 1988, X-ray emission from clusters of galaxies (Cambridge University Press)

Schellenberger, G., Reiprich, T., \& Lovisari, L. 2012, in Galaxy Clusters as Giant Cosmic Laboratories, ed. J.-U. Ness, 54

Schirmer, M. 2013, ApJS, 209, 21

Schneider, P., King, L., \& Erben, T. 2000, A\&A, 353, 41

Schrabback, T., Erben, T., Simon, P., et al. 2007, A\&A, 468, 823

Shi, X., \& Komatsu, E. 2014 [arXiv: 1401.7657]

Short, C. J., Thomas, P. A., Young, O. E., et al. 2010, MNRAS, 408, 2213

Stanek, R., Rasia, E., Evrard, A. E., Pearce, F., \& Gazzola, L. 2010, ApJ, 715, 1508

Stott, J. P., Hickox, R. C., Edge, A. C., et al. 2012, MNRAS, 422, 2213

Tremaine, S., Gebhardt, K., Bender, R., et al. 2002, ApJ, 574, 740

Vikhlinin, A., Kravtsov, A., Forman, W., et al. 2006, ApJ, 640, 691

Vikhlinin, A., Burenin, R. A., Ebeling, H., et al. 2009a, ApJ, 692, 1033 (V09a)

Vikhlinin, A., Kravtsov, A. V., Burenin, R. A., et al. 2009b, ApJ, 692, 1060

von der Linden, A., Allen, M. T., Applegate, D. E., et al. 2014a, MNRAS, 439, 2

von der Linden, A., Mantz, A., Allen, S. W., et al. 2014b [arXiv : 1402 .2670]

Weiner, B. J., Willmer, C. N. A., Faber, S. M., et al. 2006, ApJ, 653, 1049

Williams, M. J., Bureau, M., \& Cappellari, M. 2010, MNRAS, 409, 1330

Woody, D., Padin, S., Chauvin, E., et al. 2012, in SPIE Conf. Ser., 8444

Wright, C. O., \& Brainerd, T. G. 2000, ApJ, 534, 34

Zhang, Y.-Y., Finoguenov, A., Böhringer, H., et al. 2008, A\&A, 482, 451

Zhang, Y.-Y., Okabe, N., Finoguenov, A., et al. 2010, ApJ, 711, 1033 


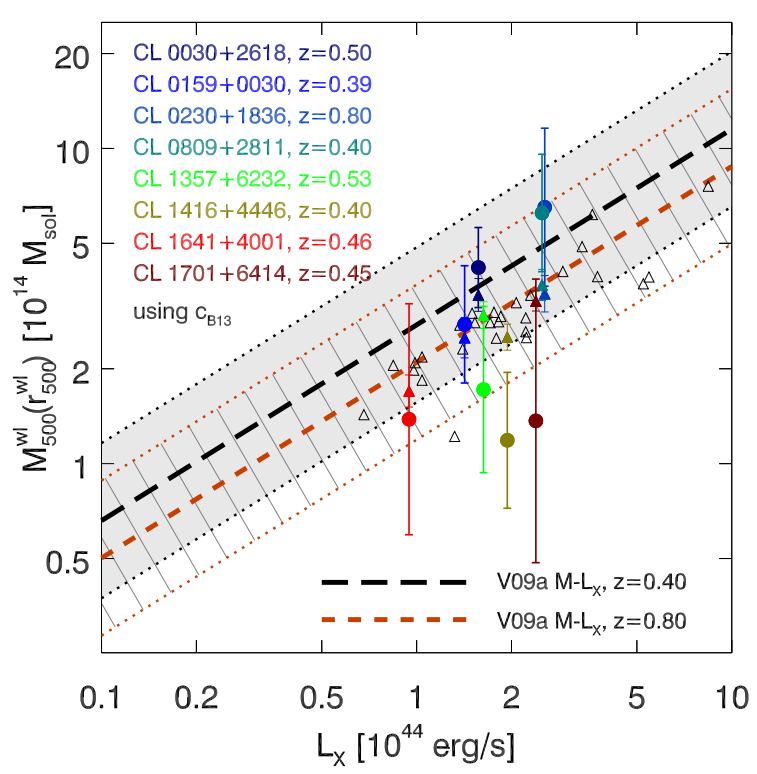

Fig. A.1. Lensing mass $-\mathrm{X}$-ray luminosity relation. The $M-L_{\mathrm{X}}$ relation is shown, for both $M_{500}^{\mathrm{wl}}\left(r_{500}^{\mathrm{wl}}\right)$ (filled circles) and $M_{500}^{\mathrm{Y}}\left(r_{500}^{\mathrm{Y}}\right)$ (small triangles). Open triangles represent the sample clusters for which MMT lensing masses are not available. The V09a $M-L_{\mathrm{X}}$ relation at $z=0.40$ $(z=0.80)$ is denoted by a long-dashed black (short-dashed red) line. Shaded (hatched) areas show the respective $1 \sigma$ intrinsic scatter ranges.

\section{Appendix A: Further scaling relations and tests}

\section{A.1. The $L_{X}-M$ relation}

To better assess the consistency of our weak lensing masses with the Vikhlinin et al. (2009a) results, we compare them to the $L_{X^{-}}$ $M_{\mathrm{Y}}$-relation derived by V09a using the $M_{500}^{\mathrm{Y}}$ masses of their low-z cluster sample. Figure A.1 inverts this relation by showing the $M_{500}^{\mathrm{wl}}\left(r_{500}^{\mathrm{wl}}\right)$ masses as a function of the $0.5-2.0 \mathrm{keV}$ Chandra luminosities measured by V09a. Statistical uncertainties in the Chandra fluxes and, hence, luminosities are negligible for our purposes. We calculate the expected $68 \%$ confidence ranges in mass for a given luminosity by inverting the scatter in $L_{X}$ at a fixed $M^{\mathrm{Y}}$ as given in Eq. (22) of V09a. For two fiducial redshifts, $z=0.40$ and $z=0.80$, spanning the unevenly populated redshift range of the eight clusters, the $M-L_{\mathrm{X}}$ relations and their expected scatter are shown in Fig. A.1. Small filled triangles in Fig. A.1 show the $M_{500}^{\mathrm{Y}}$ masses from which V09a derived the $L_{\mathrm{X}}-M$ relation. Our $8 \mathrm{MMT}$ clusters are nicely tracing the distribution of the overall sample of 36 clusters (open triangles).

As an important step in the calculation of the mass function, these authors show that their procedure is able to correct for the Malmquist bias even in the presence of evolution in the $L_{\mathrm{X}}-M$ relation, which they include in the model. We emphasise that the Malmquist bias correction - which is not included here - applied by V09a moves the clusters upwards in Fig. A.1, such that the sample agrees with the best-fit from the low- $z$ sample, as Fig. 12 in V09a demonstrates.

As already seen in Fig. 2, the $M^{\text {wl }}$ (large symbols in Fig. A.1) and $M^{\mathrm{Y}}$ agree well. Thus we can conclude that the WL masses are consistent with the expectations from their $L_{X}$. Finally, we remark that the higher X-ray luminosities for the some of the same clusters reported by Maughan et al. (2012) in their study of the $L_{X}-T_{X}$ relation are not in disagreement with V09a, as Maughan et al. (2012) used bolometric luminosities.

\section{A.2. Redshift scaling and cross-scaling of $X$-ray masses}

Here we show further results mentioned in the main body of the article. Figure A.2 shows two examples of the X-ray/WL mass ratio as a function of redshift. Owing to the inhomegenous redshift coverage of our clusters, we cannot constrain a redshift evolution. All of our bias estimates are consistent with zero bias.

Table A. 2 shows the fit results and bias estimates for various tests we performed modifying our default model, as well as for ancillary scaling relations. In particular, we probe the scaling behaviour of hydrostatic masses against the V09a estimates, for which we find a $M^{\mathrm{Y}} / M^{\text {hyd }}$ tentatively biased high by $\sim 15 \%$, while $M^{\mathrm{T}}$ and $M^{\mathrm{G}}$ do not show similar biases.

\section{A.3. Choice of centre and fitting range}

Weak lensing masses obtained from profile fitting have been shown to be sensitive to the choice of the fitting range (Becker \& Kravtsov 2011; Hoekstra et al. 2011b; Oguri \& Hamana 2011). Taking these results into account, we fitted the WL masses within a fixed physical mass range. Varying the fitting range by using $r_{\min }=0$ instead of $0.2 \mathrm{Mpc}$ in one and $r_{\max }=4.0 \mathrm{Mpc}$ instead of $5.0 \mathrm{Mpc}$ in another test, we find no evidence for a crucial influence on our results.

Both simulations and observations establish (e.g. Dietrich et al. 2012; George et al. 2012) that WL masses using lensing cluster centres are biased high due to random noise with respect to those based on independently obtained cluster centres, e.g. the ROSAT centres we employ. The fact that the $M_{500}^{\mathrm{wl}}-M_{500}^{\text {hyd }}$ relation gives slightly milder difference between $b_{\mathrm{MC}}$ for the highand low- $M^{\mathrm{wl}}$ bins when the peak of the $S$-statistics is assumed as the cluster centre (Table A.2) can be explained by the larger relative $M^{\mathrm{wl}}$ "boost" for clusters with larger offset between X-ray and lensing peaks. This affects the flat-profile clusters (Sect. 4.1) in particular, translating into a greater effect for the $c_{\text {fit }}$ case than for $c_{\mathrm{B} 13}$-based masses. We find that WL cluster centres only slightly alleviate the observed mass-dependence. 

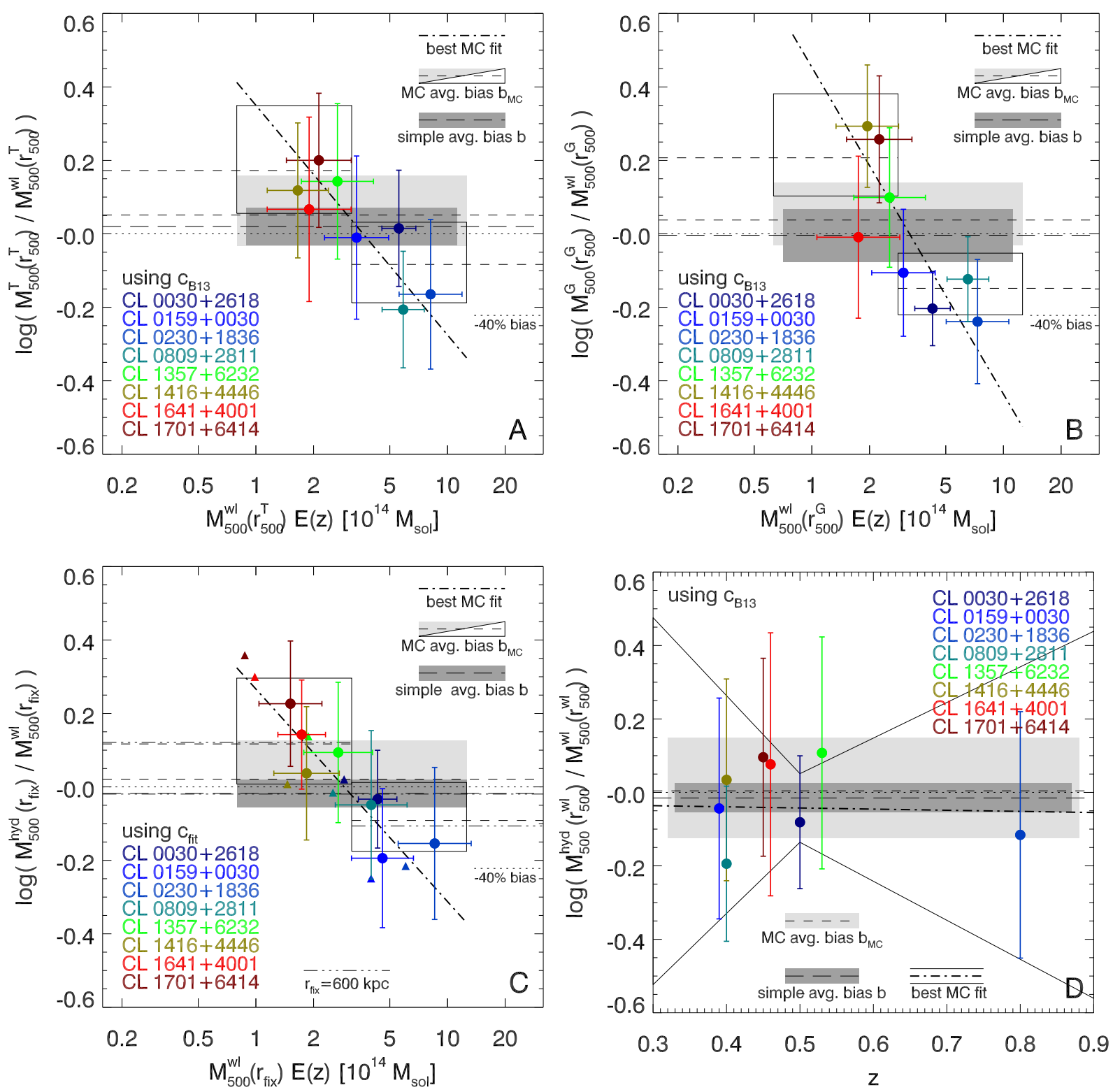

Fig. A.2. Continuation of Fig. 2. Panel A) shows $\log \left(M^{\mathrm{T}} / M^{\mathrm{wl}}\right)$ within $r_{500}^{\mathrm{T}}$, panel B) shows $\log \left(M^{\mathrm{G}} / M^{\mathrm{wl}}\right)$ within $r_{500}^{\mathrm{G}}$. Like panel A) of Fig. 2, panel C) presents $\log \left(M^{\text {hyd }} / M^{\text {wl }}\right)$, but showing both WL masses measured at a fixed physical radius $r_{\text {fix }}$. Filled dots and dot-dashed lines correspond to $r_{\text {fix }}=800 \mathrm{kpc}$, while triangles and triple-dot-dashed lines denote $r_{\text {fix }}=600 \mathrm{kpc}$. Uncertainties for the $600 \mathrm{kpc}$ case were omitted for clarity. Panel D) shows $\log \left(M^{\text {hyd }} / M^{\mathrm{wl}}\right)$ from Fig. 2 as a function of redshift. Thin solid lines indicating the $1 \sigma$ uncertainty range of the best-fit Monte Carlo/jackknife regression line (dot-dashed). 
H. Israel et al.: The 400d Galaxy Cluster Survey weak lensing programme. III.

Table A.1. Continuation of Table 1.

\begin{tabular}{|c|c|c|c|c|c|c|c|c|}
\hline & $\begin{array}{c}\text { CL } 0030 \\
+2618\end{array}$ & $\begin{array}{c}\text { CL } 0159 \\
+0030\end{array}$ & $\begin{array}{c}\text { CL } 0230 \\
+1836\end{array}$ & $\begin{array}{c}\text { CL } 0809 \\
+2811\end{array}$ & $\begin{array}{c}\text { CL } 1357 \\
+6232\end{array}$ & $\begin{array}{c}\text { CL } 1416 \\
+4446\end{array}$ & $\begin{array}{c}\text { CL } 1641 \\
+4001\end{array}$ & $\begin{array}{c}\text { CL } 1701 \\
+6414\end{array}$ \\
\hline$r_{500}^{\mathrm{wl}}\left(c_{\mathrm{B} 13}\right)$, varying $\langle\beta\rangle[\mathrm{kpc}]$ & $895_{-90}^{+84}$ & $829_{-175}^{+142}$ & $909_{-191}^{+159}$ & $1075_{-135}^{+122}$ & $710_{-161}^{+136}$ & $650_{-123}^{+104}$ & $687_{-181}^{+136}$ & $668_{-143}^{+117}$ \\
\hline$M_{500}^{\mathrm{wl}}\left(r_{500}^{\mathrm{wl}}\right)$, using $c_{\mathrm{B} 13}$, varying $\langle\beta\rangle$ & $3.70_{-1.01}^{+1.14}$ & $2.58_{-1.32}^{+1.57}$ & $5.54_{-2.81}^{+3.45}$ & $5.70_{-1.89}^{+2.17}$ & $1.91_{-1.03}^{+1.32}$ & $1.26_{-0.59}^{+0.71}$ & $1.60_{-0.96}^{+1.15}$ & $1.45_{-0.75}^{+0.90}$ \\
\hline$M^{\mathrm{wl}}\left(r_{\mathrm{fix}}=800 \mathrm{kpc}\right)$ & $3.57_{-0.76}^{+0.01}$ & $2.61_{-1.09}^{+0.92}$ & $5.33_{-2.15}^{+1.89}$ & $4.37_{-1.13}^{+1.89}$ & $1.99_{-0.94}^{+0.03}$ & $1.46_{-0.57}^{+0.59}$ & $1.66_{-0.97}^{+0.90}$ & $1.65_{-0.69}^{+0.59}$ \\
\hline$M_{500}^{\mathrm{wl}}\left(r_{500}^{\mathrm{Y}}\right)$, using $c_{\mathrm{fit}}$ & $3.71_{-0.76}^{+0.87}$ & $3.78_{-1.55}^{+1.27}$ & $5.30_{-2.15}^{+2.55}$ & $4.08_{-1.21}^{+1.91}$ & $2.08_{-0.87}^{+0.84}$ & $1.52_{-0.63}^{+0.56}$ & $1.05_{-0.27}^{+0.35}$ & $1.42_{-0.49}^{+0.55}$ \\
\hline$M_{500}^{\mathrm{wl}}\left(r_{500}^{\mathrm{Y}}\right)$, using $c_{\text {fit }}$, no dilu. corr. & $3.28_{-0.65}^{+0.72}$ & $3.43_{-1.33}^{+1.13}$ & $4.71_{-1.89}^{+2.21}$ & $3.65_{-1.04}^{+1.53}$ & - & - & - & - \\
\hline$M_{500}^{\mathrm{wl}}\left(r_{500}^{\mathrm{Y}}\right)$, using $c_{\mathrm{B} 13}$, no dilu. corr. & $3.41_{-0.73}^{+0.66}$ & $2.48_{-1.05}^{+0.86}$ & $4.67_{-1.87}^{+1.68}$ & $4.70_{-1.17}^{+1.12}$ & - & - & - & - \\
\hline$M_{500}^{\mathrm{hyd}}\left(r_{500}^{\mathrm{wl}}\right)$, using $c_{\mathrm{B} 13}$, varying $\langle\beta\rangle$ & $3.32_{-0.96}^{+1.07}$ & $2.44_{-0.99}^{+1.11}$ & $4.54_{-2.12}^{+2.57}$ & $3.86_{-1.17}^{+1.31}$ & $2.30_{-0.88}^{+0.96}$ & $1.31_{-0.45}^{+0.52}$ & $1.70_{-0.73}^{+0.73}$ & $1.82_{-0.56}^{+0.52}$ \\
\hline$M^{\text {hyd }}\left(r_{\text {fix }}=800 \mathrm{kpc}\right)$ & $3.06 \pm 0.61$ & $2.39 \pm 0.54$ & $3.84 \pm 0.71$ & $2.89 \pm 0.51$ & $2.51 \pm 0.38$ & $1.62 \pm 0.23$ & $1.88 \pm 0.35$ & $2.01 \pm 0.21$ \\
\hline$M_{500}^{\text {hyd }}\left(r_{500}^{\mathrm{Y}}\right)$ & $3.29_{-0.88}^{+0.97}$ & $2.44_{-0.77}^{+0.85}$ & $3.67_{-1.14}^{+1.34}$ & $3.41_{-0.86}^{+0.94}$ & $2.55_{-0.58}^{+0.60}$ & $1.66_{-0.42}^{+0.45}$ & $1.73_{-0.54}^{+0.56}$ & $2.15_{-0.37}^{+0.38}$ \\
\hline$M_{500}^{\text {hyd }}\left(r_{500}^{\mathrm{T}}\right)$ & $3.52_{-0.88}^{+0.95}$ & $2.48_{-0.76}^{+0.84}$ & $3.73_{-1.14}^{+1.34}$ & $3.16_{-0.89}^{+0.98}$ & $2.52_{-0.59}^{+0.61}$ & $1.47_{-0.46}^{+0.52}$ & $1.74_{-0.54}^{+0.56}$ & $2.04_{-0.40}^{+0.41}$ \\
\hline$M_{500}^{\text {hyd }}\left(r_{500}^{\mathrm{G}}\right)$ & $2.83_{-0.93}^{+1.05}$ & $2.26_{-0.79}^{+0.90}$ & $3.21_{-1.13}^{+1.38}$ & $3.49_{-0.86}^{+0.93}$ & $2.42_{-0.62}^{+0.64}$ & $1.77_{-0.40}^{+0.42}$ & $1.64_{-0.60}^{+0.61}$ & $2.13_{-0.37}^{+0.39}$ \\
\hline
\end{tabular}

Notes. Further measured properties of the 400d MMT cluster sample. All masses are in units of $10^{14} M_{\odot}$, without applying the $E(z)$ factor. We state only stochastic uncertainties, i.e. do not include systematics. By $c_{\mathrm{fit}}$ and $c_{\mathrm{B} 13}$ we denote the choices for the NFW concentration parameter explained in Sect. 2.2. We refer to Sect. 4.3 for the introduction of the "varying $\langle\beta\rangle$ " case.

Table A.2. Continuation of Table 2.

\begin{tabular}{|c|c|c|c|c|c|c|c|c|}
\hline Scaling relation & Model & $c_{\mathrm{NFW}}$ & Slope $B$ & Intercept $A$ & $b_{\mathrm{MC}}$ from Monte Carlo & $b=\langle\log \xi-\log \eta\rangle$ & $\chi_{\mathrm{red}, \mathrm{M}-\mathrm{M}}^{2}$ & Section \\
\hline \multirow[t]{4}{*}{$M_{500}^{\mathrm{wl}}\left(r_{500}^{\mathrm{wl}}\right)-M_{500}^{\mathrm{hyd}}\left(r_{500}^{\mathrm{wl}}\right)$} & varying $\langle\beta\rangle$ & $c_{\text {fit }}$ & $-0.54_{-0.21}^{+0.22}$ & $0.00_{-0.08}^{+0.07}$ & $0.08_{-0.13}^{+0.14}\left(0.23_{-0.18}^{+0.20} ;-0.07_{-0.15}^{+0.16}\right)$ & $0.07 \pm 0.07$ & 0.60 & 4.3 \\
\hline & varying $\langle\beta\rangle$ & $c_{\mathrm{B} 13}$ & $-0.50_{-0.30}^{+0.30}$ & $-0.02 \pm 0.08$ & $0.01_{-0.13}^{+0.15}\left(0.08_{-0.18}^{+0.22} ;-0.07_{-0.15}^{+0.17}\right)$ & $-0.01 \pm 0.03$ & 0.50 & 4.3 \\
\hline & $S$-peak centred & $c_{\text {fit }}$ & $-0.48_{-0.23}^{+0.24}$ & $-0.02 \pm 0.07$ & $-0.03_{-0.11}^{+0.13}\left(0.08_{-0.16}^{+0.19} ;-0.14_{-0.14}^{+0.15}\right)$ & $-0.04 \pm 0.04$ & 0.59 & A. 3 \\
\hline & $S$-peak centred & $c_{\mathrm{B} 13}$ & $-0.47_{-0.25}^{+0.26}$ & $-0.03 \pm 0.07$ & $-0.05_{-0.10}^{+0.12}\left(0.01_{-0.14}^{+0.16} ;-0.12_{-0.14}^{+0.15}\right)$ & $-0.07 \pm 0.03$ & 0.60 & A. 3 \\
\hline \multirow[t]{3}{*}{$M_{500}^{\mathrm{wl}}\left(r_{500}^{\mathrm{Y}}\right)-M_{500}^{\mathrm{Y}}\left(r_{500}^{\mathrm{Y}}\right)$} & default & $c_{\text {fit }}$ & $-0.73_{-0.14}^{+0.13}$ & $0.05 \pm 0.04$ & $0.08_{-0.06}^{+0.08}\left(0.26_{-0.08}^{+0.11} ;-0.10_{-0.08}^{+0.10}\right)$ & $0.06 \pm 0.07$ & 1.67 & 3.2 \\
\hline & no dilu. corr. & $c_{\text {fit }}$ & $-0.71_{-0.15}^{+0.14}$ & $0.06 \pm 0.04$ & $0.11_{-0.06}^{+0.08}\left(0.26_{-0.08}^{+0.11} ;-0.05_{-0.08}^{+0.10}\right)$ & $0.09 \pm 0.06$ & 1.51 & 4.3 \\
\hline & no dilu. corr. & $c_{\mathrm{B} 13}$ & $-0.73_{-0.15}^{+0.13}$ & $0.07 \pm 0.03$ & $0.10_{-0.07}^{+0.10}\left(0.23_{-0.11}^{+0.18} ;-0.04_{-0.07}^{+0.10}\right)$ & $0.06 \pm 0.05$ & 1.08 & 4.3 \\
\hline$M_{500}^{\mathrm{wl}}\left(r_{500}^{\mathrm{Y}}\right)-M_{500}^{\mathrm{hyd}}\left(r_{500}^{\mathrm{Y}}\right)$ & default & $c_{\text {fit }}$ & $-0.63_{-0.20}^{+0.20}$ & $0.01 \pm 0.05$ & $0.02_{-0.08}^{+0.09}\left(0.14_{-0.10}^{+0.12} ;-0.12_{-0.11}^{+0.12}\right)$ & $0.01 \pm 0.05$ & 0.77 & 3.2 \\
\hline$M_{500}^{\mathrm{wl}}\left(r_{500}^{\mathrm{Y}}\right)-M_{500}^{\mathrm{hyd}}\left(r_{500}^{\mathrm{Y}}\right)$ & default & $c_{\mathrm{B} 13}$ & $-0.56_{-0.23}^{+0.23}$ & $0.00 \pm 0.05$ & $0.02_{-0.09}^{+0.11}\left(0.12_{-0.12}^{+0.18} ;-0.10_{-0.10}^{+0.12}\right)$ & $-0.02 \pm 0.04$ & 0.65 & 3.2 \\
\hline$M_{500}^{\mathrm{wl}}\left(r_{500}^{\mathrm{T}}\right)-M_{500}^{\mathrm{hyd}}\left(r_{500}^{\mathrm{T}}\right)$ & default & $c_{\mathrm{B} 13}$ & $-0.53_{-0.22}^{+0.23}$ & $0.04 \pm 0.05$ & $0.03_{-0.05}^{+0.06}\left(0.09_{-0.06}^{+0.07} ;-0.05_{-0.08}^{+0.09}\right)$ & $-0.02 \pm 0.04$ & 0.66 & 3.2 \\
\hline$M_{500}^{\mathrm{wl}}\left(r_{500}^{\mathrm{G}}\right)-M_{500}^{\mathrm{hyd}}\left(r_{500}^{\mathrm{G}}\right)$ & default & $c_{\mathrm{B} 13}$ & $-0.61_{-0.25}^{+0.24}$ & $-0.01 \pm 0.06$ & $0.01_{-0.09}^{+0.11}\left(0.12_{-0.12}^{+0.18} ;-0.10_{-0.12}^{+0.12}\right)$ & $-0.02 \pm 0.04$ & 0.62 & 3.2 \\
\hline$M_{500}^{\text {hyd }}\left(r_{500}^{\mathrm{Y}}\right)-M_{500}^{\mathrm{Y}}\left(r_{500}^{\mathrm{Y}}\right)$ & default & - & $-0.76_{-0.29}^{+0.21}$ & $0.07 \pm 0.03$ & $0.07 \pm 0.05$ & $0.06 \pm 0.03$ & 1.29 & 3.2 \\
\hline$M_{500}^{\text {hyd }}\left(r_{500}^{\mathrm{T}}\right)-M_{500}^{\mathrm{T}}\left(r_{500}^{\mathrm{T}}\right)$ & default & - & $-0.53_{-0.39}^{+0.37}$ & $0.04 \pm 0.05$ & $0.04 \pm 0.07$ & $0.04 \pm 0.02$ & 0.79 & 3.2 \\
\hline$M_{500}^{\text {hyd }}\left(r_{500}^{\mathrm{G}}\right)-M_{500}^{\mathrm{G}}\left(r_{500}^{\mathrm{G}}\right)$ & default & - & $-1.20_{-0.62}^{+0.36}$ & $-0.05_{-0.04}^{+0.03}$ & $0.03_{-0.05}^{+0.06}$ & $0.01 \pm 0.05$ & 1.85 & 3.2 \\
\hline \multirow[t]{2}{*}{$M_{500}^{\mathrm{Y}}\left(r_{500}^{\mathrm{Y}}\right)-M_{500}^{\mathrm{T}}\left(r_{500}^{\mathrm{T}}\right)$} & default (MMT8) & - & $-0.07_{-0.51}^{+0.65}$ & $-0.03_{-0.07}^{+0.06}$ & $-0.04 \pm 0.05$ & $-0.03 \pm 0.03$ & 1.27 & 4.4 \\
\hline & all 36 & - & $-0.05_{-0.12}^{+0.13}$ & $0.00 \pm 0.02$ & $-0.03 \pm 0.02$ & $-0.02 \pm 0.01$ & 1.32 & 4.4 \\
\hline \multirow[t]{2}{*}{$M_{500}^{\mathrm{Y}}\left(r_{500}^{\mathrm{Y}}\right)-M_{500}^{\mathrm{G}}\left(r_{500}^{\mathrm{G}}\right)$} & default (MMT8) & - & $-0.39_{-0.55}^{+0.47}$ & $-0.02_{-0.04}^{+0.05}$ & $-0.07 \pm 0.02$ & $-0.07 \pm 0.04$ & 3.43 & 4.4 \\
\hline & all 36 & - & $-0.05_{-0.06}^{+0.06}$ & $-0.05 \pm 0.01$ & $-0.07 \pm 0.01$ & $-0.07 \pm 0.01$ & 2.32 & 4.4 \\
\hline \multirow[t]{2}{*}{$M_{500}^{\mathrm{T}}\left(r_{500}^{\mathrm{T}}\right)-M_{500}^{\mathrm{G}}\left(r_{500}^{\mathrm{G}}\right)$} & default (MMT8) & - & $-1.24_{-0.41}^{+0.28}$ & $0.05 \pm 0.03$ & $-0.03_{-0.04}^{+0.05}$ & $-0.04 \pm 0.06$ & 2.81 & 4.4 \\
\hline & all 36 & - & $-0.55_{-0.14}^{+0.10}$ & $0.02 \pm 0.02$ & $-0.04 \pm 0.02$ & $-0.05 \pm 0.02$ & 2.58 & 4.4 \\
\hline
\end{tabular}

Notes. We estimate a possible bias between masses $\xi$ and $\eta$ by three estimators: First, we fit to $(\log \xi-\log \eta$ ) as a function of $\eta$, yielding an intercept $A$ at pivot $\log \left(M_{\text {piv }} / M_{\odot}\right)=14.5$ and slope $B$ from the Monte Carlo/jackknife analysis. Second, we compute the logarithmic bias $b_{\mathrm{MC}}=\langle\log \xi-\log \eta\rangle_{\mathrm{MC}}$, averaged over the same realisations. Uncertainties for the MC results are given by $1 \sigma$ ensemble dispersions. In parentheses next to $b_{\mathrm{MC}}$, we show its value for the low- $M^{\text {wl }}$ and high- $M^{\text {wl }}$ clusters. Third, we quote the logarithmic bias $b=\langle\log \xi-\log \eta\rangle$ obtained directly from the input masses, along with its standard error. Finally, we give the $\chi_{\text {red }}^{2}$ for the mass-mass scaling, obtained from the MC method. The "default" model denotes WL and hydrostatic masses as described in Sect. 2. 